\title{
BOUNDS FOR THE FIRST DIRICHLET EIGENVALUE OF TRIANGLES AND QUADRILATERALS
}

\author{
Pedro Freitas $^{1}$ And Bateomiej Siudeja ${ }^{2}$
}

\begin{abstract}
We prove some new upper and lower bounds for the first Dirichlet eigenvalue of triangles and quadrilaterals. In particular, we improve Pólya and Szegö's [Annals of Mathematical Studies 27 (1951)] lower bound for quadrilaterals and extend Hersch's [Z. Angew. Math. Phys. 17 (1966) 457-460] upper bound for parallelograms to general quadrilaterals.
\end{abstract}

Mathematics Subject Classification. 35P15, 35J05.

Received September 18, 2008. Revised March 5, 2009.

Published online July 2nd, 2009.

\section{INTRODUCTION}

Quadrilaterals in the Euclidean plane include one of the very few planar domains for which it is possible to evaluate the Dirichlet spectrum explicitly, namely, rectangles. In spite of this, and as far as we are aware, there is only one lower bound in the literature for the first Dirichlet eigenvalue of a general quadrilateral which takes into account its specific form. This is the 1951 bound of Pólya and Szegö's which was obtained in the process of proving that of all planar quadrilaterals of equal area, the square minimizes the first Dirichlet eigenvalue [10] (we note in passing that the general result conjectured in [10], namely, that of all $n$-polygons of fixed area the regular polygon minimizes the first Dirichlet eigenvalue remains open to this day for $n$ larger than four). The analytic version of this statement is

$$
\frac{2 \pi^{2}}{A} \leq \lambda_{1}(Q)
$$

where $A$ denotes the area of the quadrilateral $Q$ and $\lambda_{1}$ its first Dirichlet eigenvalue - this notation will be used throughout the paper for triangles, rhombi and general quadrilaterals. Such a bound is what is referred to in [2] as static, as it does not take into consideration the deviation from the optimizer. There are, of course, lower bounds such as that of Makai [8] for general convex domains which measure this deviation by means of the perimeter or the diameter. However, these do not take advantage of the specific class of domains considered, since they are valid within the larger class of convex domains. On the other hand, recent work has shown that there is still much room for improvement in this area both for specific domains $[4,5,13,14]$ and also in more

\footnotetext{
Keywords and phrases. Dirichlet eigenvalues, polygons, variational bounds.

1 Department of Mathematics, Faculdade de Motricidade Humana (TU Lisbon) and Group of Mathematical Physics of the

University of Lisbon, Complexo Interdisciplinar, Av. Prof. Gama Pinto 2, 1649-003 Lisboa, Portugal. freitas@cii.fc.ul.pt

2 Department of Mathematics, University of Illinois at Urbana-Champaign, Urbana, IL 61801, USA. siudeja@illinois.edu
} 
general settings [3]. Furthermore, extensive numerical simulation has also pointed to new connections between spectral quantities such as the first Dirichlet eigenvalue or the spectral gap and simple geometric quantities [1,2].

The purpose of the present paper is twofold. On one hand, we continue the work in the papers mentioned above and, by combining several of the techniques introduced previously, provide a collection of improved bounds for triangles and rhombi. On the other hand, we apply these techniques to the case of quadrilaterals, thus obtaining a set of upper and lower bounds in this instance. As an example of our results, we state here the main result for quadrilaterals:

Theorem 1.1. Let $Q$ be a quadrilateral with side lengths $\ell_{i}, i=1, \ldots, 4$, and diagonals of lengths $d_{1}$ and $d_{2}$ forming an angle $\theta$ in $(0, \pi / 2]$ between them, where it is assumed that $d_{2}$ is the length of the largest diagonal contained in $Q$. Then

$$
\frac{2 \pi^{2}}{A}+\frac{\pi^{2}}{4 A^{2}}\left(d_{2} \sin \theta-d_{1}\right)^{2} \leq \lambda_{1}(Q) \leq \frac{\pi^{2}}{2 A^{2}}\left(l_{1}^{2}+l_{2}^{2}+l_{3}^{2}+l_{4}^{2}\right),
$$

where $A$ denotes the area of $Q$. Equality holds for squares and rectangles in the lower and upper bounds respectively.

Remark 1.2. Since we have $l_{1}^{2}+l_{2}^{2}+l_{3}^{2}+l_{4}^{2}=d_{1}^{2}+d_{2}^{2}+4 m^{2}$, where $m$ is the length of the segment joining the middle points of the two diagonals, and $A=\frac{1}{2} d_{1} d_{2} \sin \theta$, both bounds may actually be written in terms of the diagonal lengths, the angle formed by them and, in the case of the upper bound, also of $m$

Remark 1.3. In [1] it was conjectured that

$$
\lambda_{1}(Q) \leq \pi^{2} \frac{L^{2}-8 A}{4 A^{2}},
$$

where $L$ denotes the perimeter. Although the upper bound in the above theorem also yields equality for rectangles, it is not strong enough to show the conjecture holds in general - it is worse for degenerate quadrilaterals (in fact, isosceles triangles) with side lengths $0, b, \ell, \ell$ satisfying $5 b>8 \ell$, for instance. On the other hand, it is better than (or at least as good as) the conjecture for a class of quadrilaterals which includes parallelograms.

Remark 1.4. The lower bound includes Pólya and Szegö's bound plus a correction term, thus making it an improvement of (1.1).

The upper bound in Theorem 1.1 had been conjectured in [1] and it is in fact an extension to general quadrilaterals of an upper bound obtained by Hersch for parallelograms in [6]. The lower bound for quadrilaterals is a straightforward consequence of Steiner symmetrization and Hooker and Protter's lower bound for rhombi [7], but we could not find it in the literature. In a similar fashion, it is possible to combine other lower bounds for the first eigenvalue of rhombi with Steiner symmetrization, and we do this in Theorem 4.2 below where we use a bound from [5].

Throughout the paper we make a distinction between bounds which depend explicitly on simple geometric quantities, such as lengths and angles, and also possibly on zeros of Bessel functions, and those requiring either integrations or the calculation of zeros of more complicated functions. For lack of a better notation, we refer to the former as explicit bounds and to the latter as functional bounds.

The plan of the paper is as follows. In the next section we present the known bounds for triangles, together with improvements based on the combination of techniques from $[5,14]$. Section 3 contains a similar analysis, but now for rhombi. This consists mainly of new upper bounds, one of which (Thm. 3.2) is an improvement of a result from [7] which coincides with the more general upper bound in Theorem 1.1. Finally, Section 4 contains the new results for general quadrilaterals mentioned above. In the Appendices we describe and collect some of the Mathematica scripts used to compute the Rayleigh quotients for test functions obtained from known eigenfunctions. 


\section{BOUnds FOR TRIANGLES}

In this section we present the best bounds for eigenvalues of triangles. Here the angle $\alpha$ denotes the smallest angle of a triangle, $d$ its diameter, $\rho$ its inradius, $A$ its area and $L$ its perimeter. Let also $a \leq b \leq c=d$ be the lengths of the sides and $h$ denote the altitude perpendicular to the longest side.

\subsection{Explicit bounds}

We first collect upper and lower bounds for triangles which depend on simple geometric quantities (area, perimeter, etc.), and maybe zeros of Bessel functions. The following upper bound for triangles was proved by the second author in [13]

$$
\lambda_{1}(T) \leq \frac{\pi^{2} L^{2}}{9 A^{2}}=\frac{4 \pi^{2}}{9 \rho^{2}} .
$$

This is a very simple expression which gives equality for equilateral triangles. In spite of the fact that it includes the dependence on both area and perimeter, in order to obtain a bound that behaves well for thin triangles one has to consider a different point of view and include the information of the eigenvalue of a related circular sector.

Theorem 2.1. Let $\beta=\arcsin \left(2 A / d^{2}\right)=\arcsin (h / d)$ and denote the circular sector with radius $r$ and angle $\gamma$ by $S(r, \gamma)$. Then

$$
\lambda_{1}(T) \leq \lambda_{1}(S(\sqrt{A / \tan (\beta / 2)}, \beta))=\frac{j_{\pi / \beta}^{2}}{A} \tan (\beta / 2) .
$$

There are also good lower bounds for triangles which satisfy the simplicity criteria mentioned above. The first is due to Pólya and Szegö, namely,

$$
\frac{4 \pi^{2}}{\sqrt{3} A} \leq \lambda_{1}(T)
$$

and may be understood as a version of Faber-Krahn for triangles. It has the disadvantage that by depending on the area alone, it does not take into account how far one is from the optimal given by the equilateral triangle.

The first author proved in [5] the following bound

$$
\pi^{2}\left(\frac{4}{d^{2}}+\frac{1}{h^{2}}\right)=\pi^{2}\left(\frac{4}{d^{2}}+\frac{d^{2}}{4 A^{2}}\right) \leq \lambda_{1}(T) .
$$

This already contains a measure of how far we are from the equilateral triangle as it now depends also on the diameter while still giving equality for equilateral triangles. Unfortunately this bound is weaker than (2.3) for almost equilateral triangles.

Another bound was obtained by the second author in [14]

$$
\pi^{2}\left(\frac{4}{d^{2}+h^{2}}+\frac{1}{d^{2}}+\frac{1}{h^{2}}\right) \leq \lambda_{1}(T) .
$$

The maximum between this and (2.3) is always better than the previous bound. The trade-off is that (2.5) no longer yields equality for equilateral triangles.

Corollary 4.1 below provides another bound which is stronger than (2.5), but which does not give equality for equilateral triangles either:

$$
\pi^{2}\left(\frac{1}{d}+\frac{1}{h}\right)^{2}=\frac{\pi^{2}}{4 A^{2}}\left(d+\frac{2 A}{d}\right)^{2} \leq \lambda_{1}(T)
$$


The next bound is based on a bound by Protter for general convex domains - see [4,11] and further improvement made by Méndez-Hernández [9]

$$
\frac{\pi^{2}}{4}\left[\frac{1}{(d-\rho)^{2}}+\frac{1}{\rho^{2}}\right] \leq \lambda_{1}(T) .
$$

Again this bound does not give equality for equilateral triangles, but it was the best for certain narrow triangles. Corollary 4.1 is the first bound not containing the inradius which is stronger than the above (by comparing numerical results).

The second author also proved in [14] a bound similar to that in Theorem 2.1, namely,

$$
\frac{\alpha j_{\pi / \alpha}^{2}}{2 A}=\lambda_{1}(S(\alpha, A)) \leq \lambda_{1}(T),
$$

where $S(\alpha, A)$ is a circular sector with angle $\alpha$ and the same area as a given triangle.

We can also generalize [5], Proposition 2.4, to get another bound based on circular sectors.

$$
\frac{4\left(j_{\pi / \gamma}^{\prime}\right)^{2}}{4 h^{2}+d^{2}}=\frac{4\left(j_{\pi / \gamma}^{\prime}\right)^{2}}{4 b^{2} \sin ^{2} \alpha+c^{2}} \leq \lambda_{1}(T),
$$

where $\gamma=\arctan (2 b \sin \alpha / c)=\arctan (2 h / d)$.

The following theorem provides further improvement to the above bound.

Theorem 2.2. Let $\gamma=2 \arctan (b \sin \alpha / c)=2 \arctan (h / d)$. We have

$$
\frac{4\left(j_{\pi / \gamma}^{\prime}\right)^{2}}{h^{2}+d^{2}}=\frac{4\left(j_{\pi / \gamma}^{\prime}\right)^{2}}{b^{2} \sin ^{2} \alpha+c^{2}} \leq \lambda_{1}(T)
$$

It is remarkable that neither of the lower bounds above is better than Pólya's isoperimetric bound (2.3) for triangles close to equilateral triangles. The following conjecture, based on the concept of isoperimetric correction (see [1]), would provide an improvement in this range.

Conjecture 2.3 ([13], Conjecture 1.2).

$$
\frac{\pi^{2} L^{2}}{16 A^{2}}+\frac{7 \sqrt{3} \pi^{2}}{12 A} \leq \lambda_{1}(T) \leq \frac{\pi^{2} L^{2}}{12 A^{2}}+\frac{\sqrt{3} \pi^{2}}{3 A} .
$$

The upper bound would also provide a significant improvement over (2.1).

Before we proceed to functional bounds, we will compare the explicit bounds given above. All triangles (up to scaling) can be parameterized by the lengths of the sides $a=1,1 \leq b \leq c \leq b+1$. By taking $e=c-b$ the parameter space becomes $b \geq 1$ and $0 \leq e \leq 1$.

Upper bounds are compared in Figure 1. We can see that the sector based bound (Thm. 2.1) is the best in extreme cases, while the bound given by (2.1) is the best near the equilateral triangle. It is worth noting that the lower bound in Conjecture 2.3 would provide a significant improvement to (2.1), as may be seen from the comparison of lower bounds in Figure 2. In particular, it would supersede the bounds (2.3) and (2.6).

It is interesting to see how far upper bounds are from lower bounds. The following figures present this difference for certain classes of triangles. Let us divide the class of isosceles triangles into superequilateral triangles (with sides of lengths $a=b<c$ ) and subequilateral (with sides of lengths $a=b>c$ ). This is more appropriate than acute/obtuse in this context due to the extremal properties of equilateral triangles and, in fact, the study of the spectral gap in [2] had already prompted such a division. There subequilateral and superequilateral were referred to as triangles of type I and II, respectively. Figure 3 contains results for subequilateral triangles $(a=1$ and $b=c)$, Figure 4 for superequilateral triangles $(a=b=1)$, Figure 5 


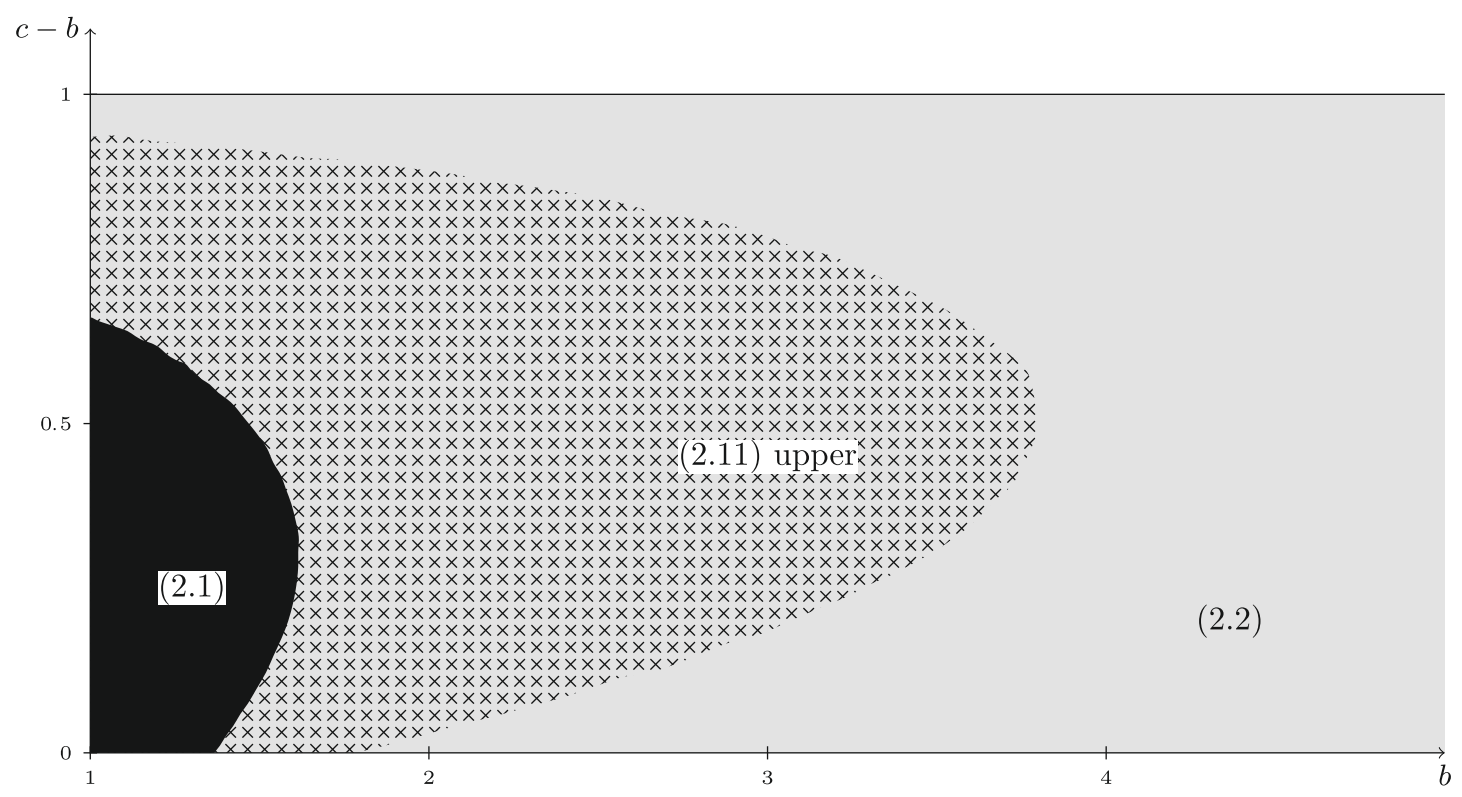

Figure 1. The best upper bounds for triangles parameterized by the lengths of the sides $1 \leq b \leq c$ (bounds (2.1) and (2.2)). Also, the crosshatched region shows where the conjectured upper bound (2.11) would provide an improvement (making (2.1) obsolete).

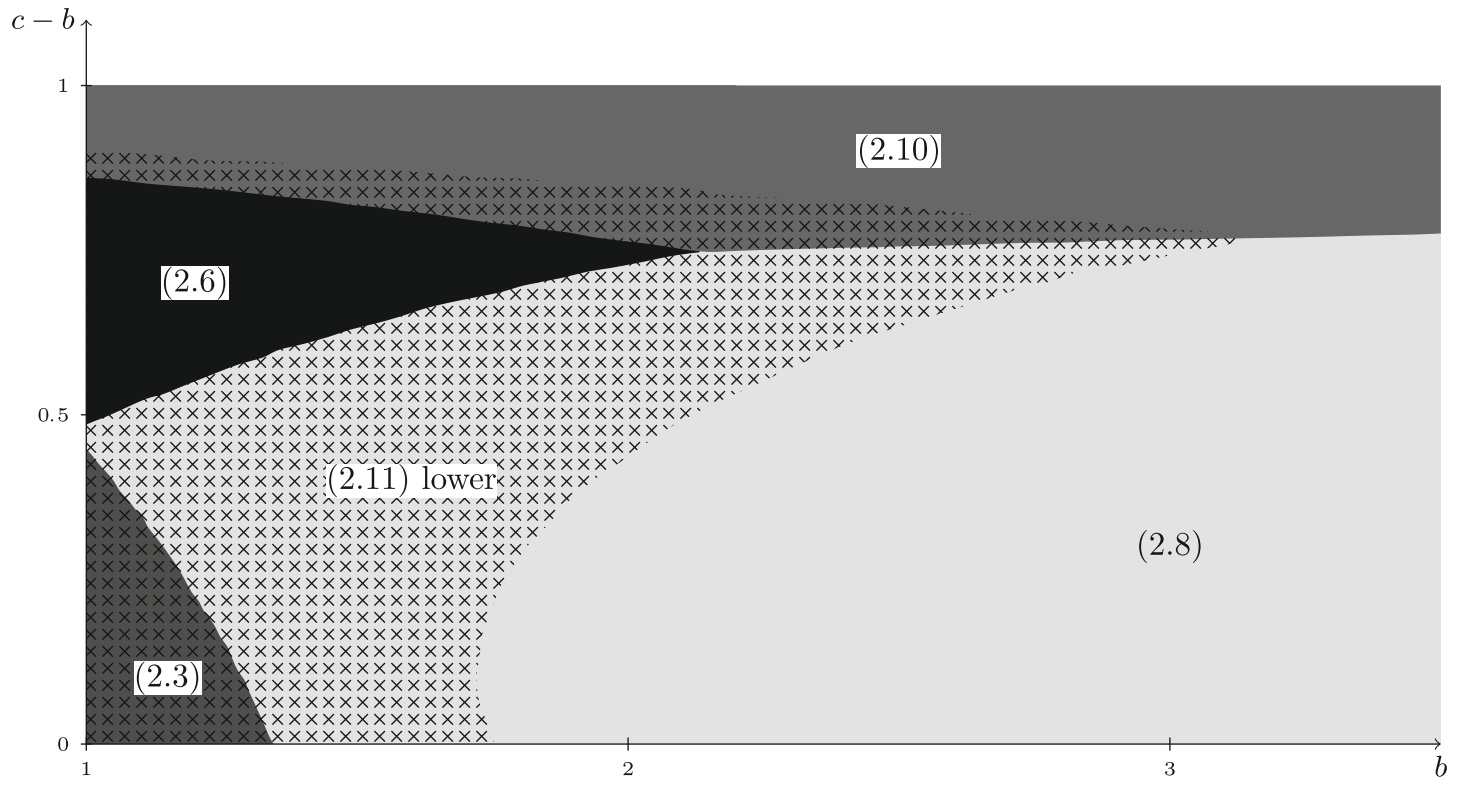

Figure 2. The best lower bounds for triangles parameterized by the lengths of the sides $1 \leq b \leq c$ (bounds (2.3), (2.6), (2.8) and (2.10)). The crosshatched region shows where the conjectured lower bound (2.11) would provide an improvement (making (2.3) and (2.6) obsolete). 


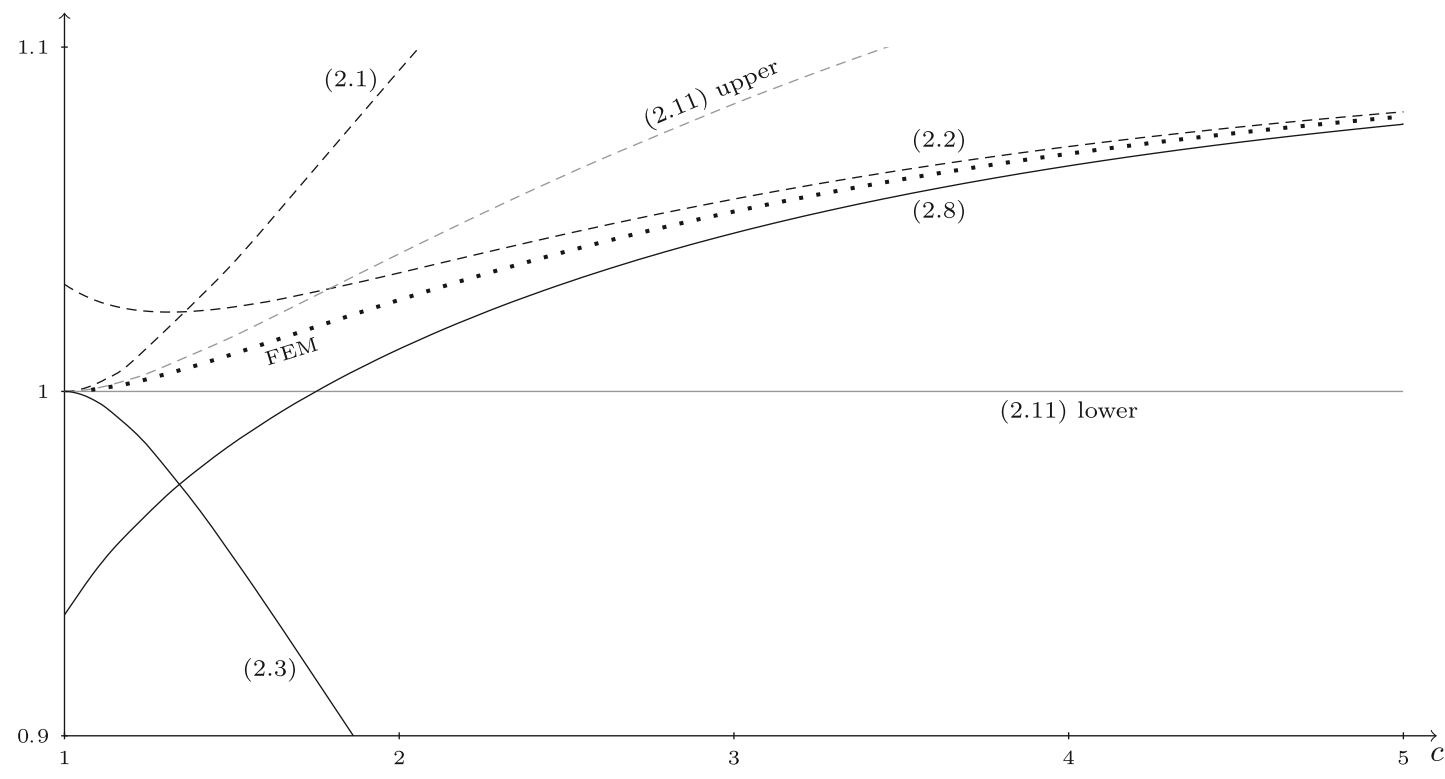

Figure 3. Comparison of lower bounds (solid curves) and upper bounds (dashed curves) for subequilateral triangles (with side lengths $1=a \leq b=c$ ). Each curve represents the ratio of the indicated bound over the conjectured lower bound (2.11) (Conjecture 2.3). The thick dotted line indicates numerical results (FEM with $2^{20}$ triangles).

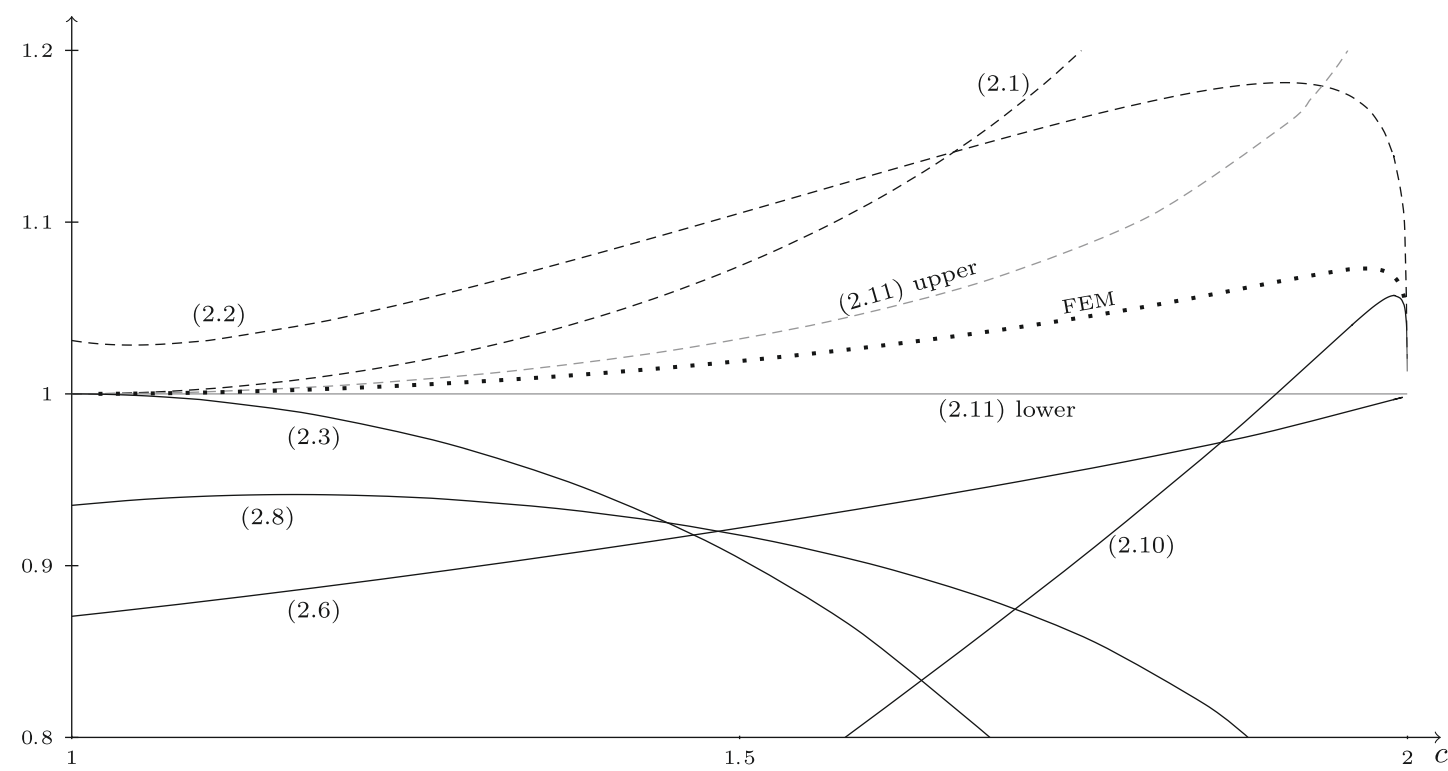

Figure 4. Comparison of lower bounds (solid curves) and upper bounds (dashed curves) for superequilateral triangles (with side lengths $1=a=b \leq c$ ). Each curve represents the ratio of the indicated bound over the conjectured lower bound (2.11) (Conjecture 2.3). The thick dotted line indicates numerical results (FEM with $2^{20}$ triangles). 


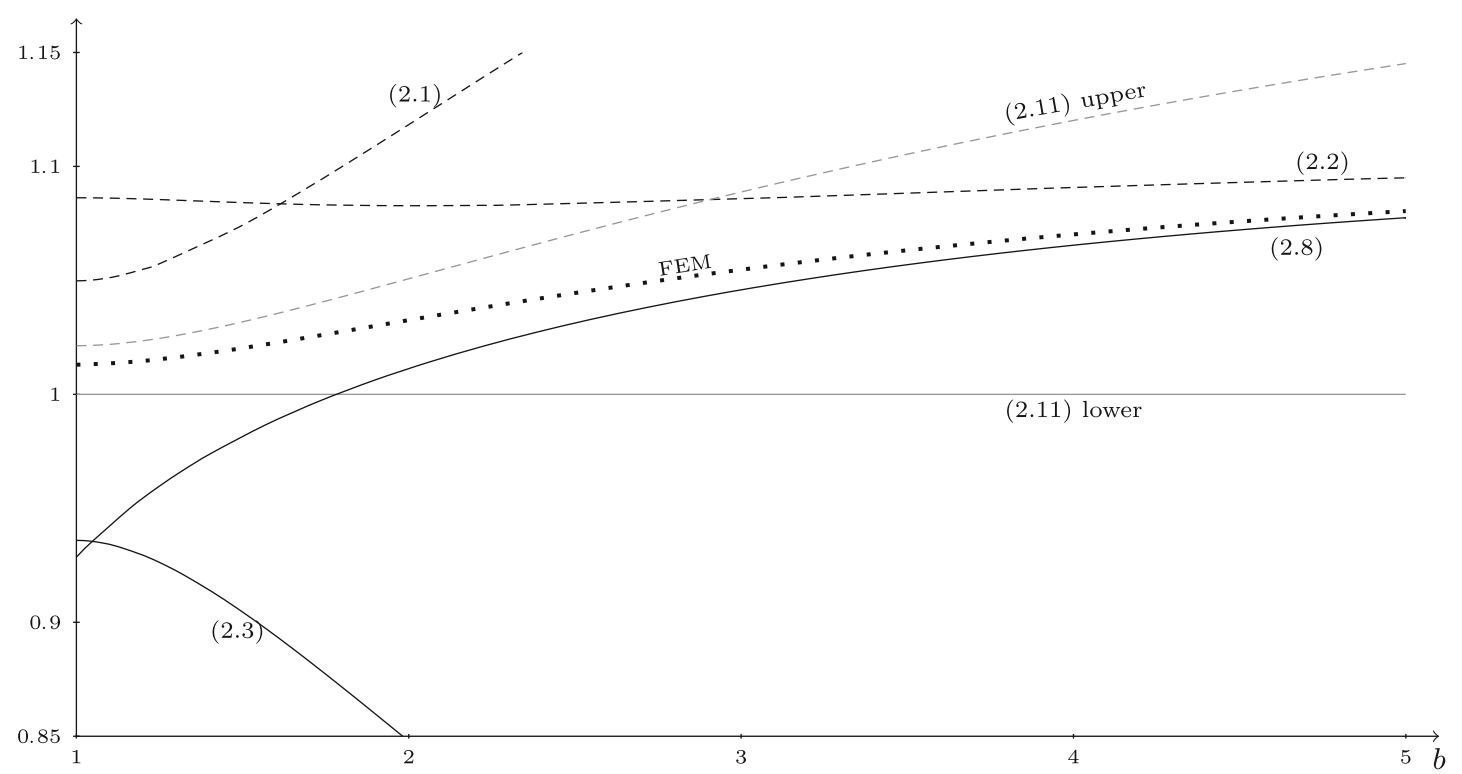

Figure 5. Comparison of lower bounds (solid curves) and upper bounds (dashed curves) for right triangles with side lengths $1 \leq b$ and $c=\sqrt{1+b^{2}}$. Each curve represents the ratio of the indicated bound over the conjectured lower bound (2.11) (Conjecture 2.3). The thick dotted line indicates numerical results (FEM with $2^{20}$ triangles).

for right triangles $\left(a=1\right.$ and $\left.c=\sqrt{b^{2}+1}\right)$ and Figure 6 for obtuse non-isosceles triangles $(a=1$ and $c=b+0.8)$. Clearly, this last case is the worst with respect to accuracy, as are non-extremal cases in other figures. This reflects the fact that all bounds are based on extremal cases.

\subsection{Functional bounds}

Let us begin with the lower bounds obtained by the first author in [5] and their variations based on symmetrization. The first of these is very good for triangles which are close to subequilateral triangles [5], Theorem 2,

$$
j_{\pi / \alpha}^{2}\left[\int_{0}^{1} \frac{2 \sin ^{2}(\pi t)}{[g(\alpha t)]^{2(\pi+\alpha) / \alpha}} \mathrm{d} t\right]^{-\alpha /(\pi+\alpha)} \leq \lambda_{1}(T)
$$

where

$$
g(\theta)=\frac{\sin (\alpha-\theta)}{c \sin \alpha}+\frac{\sin \theta}{b \sin \alpha}
$$

This bound can be improved using symmetrization. We have

Theorem 2.4. The bound (2.12) holds with the function $g(\theta)$ replaced by

$$
g_{2}(\theta)=\frac{\sin (\alpha-\theta)+\sin \theta}{\sqrt{b c} \sin \alpha},
$$




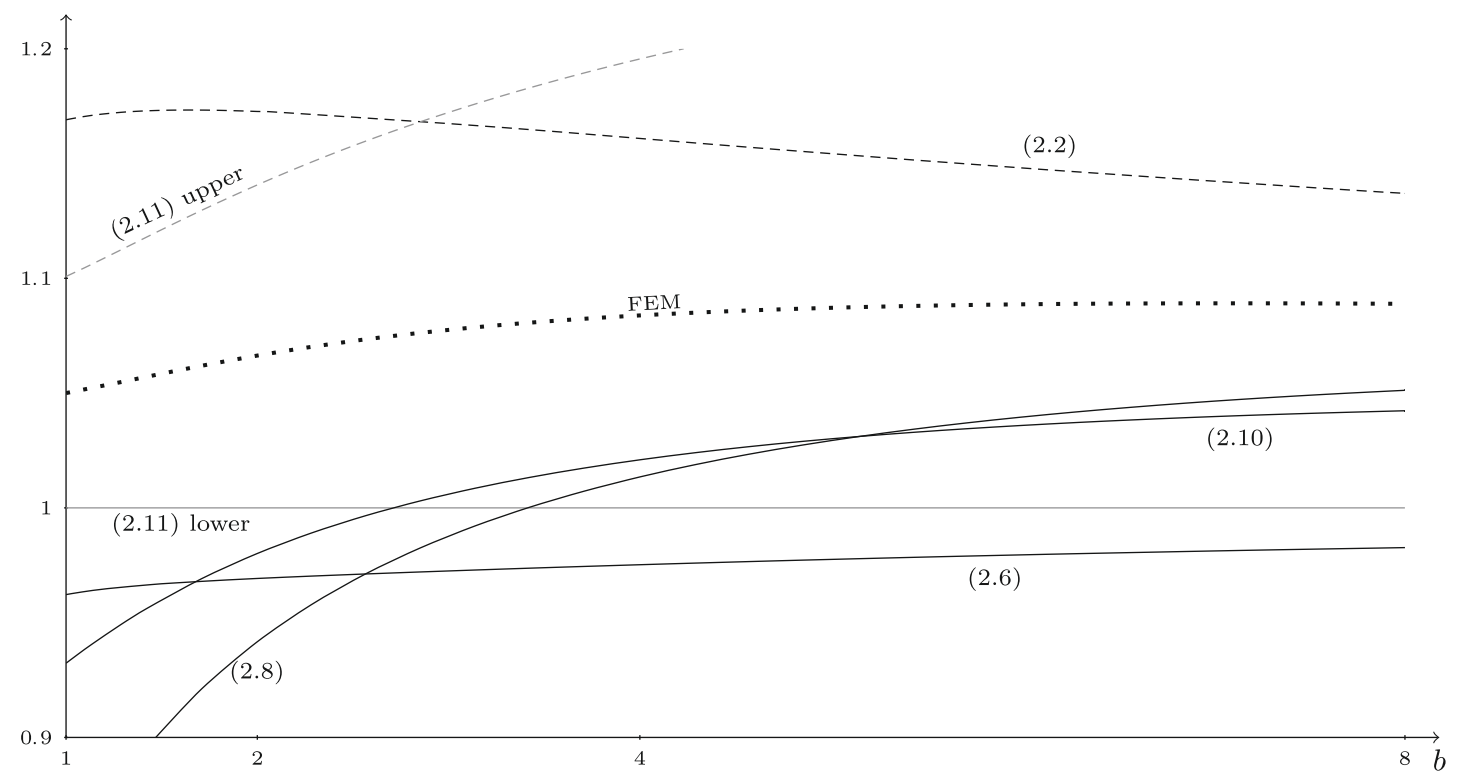

Figure 6. Comparison of lower bounds (solid curves) and upper bounds (dashed curves) for some obtuse non-isosceles triangles with side lengths $1 \leq b$ and $c=b+0.8$. Each curve represents the ratio of the indicated bound over the conjectured lower bound (2.11) (Conjecture 2.3). The thick dotted line indicates numerical results (FEM with $2^{20}$ triangles).

yielding

$$
\frac{j_{\pi / \alpha}^{2}}{4 A} \tan (\alpha / 2)\left[\int_{0}^{1} \frac{2 \sin ^{2}(\pi t)}{[2 \cos (\alpha / 2-\alpha t)]^{2(\pi+\alpha) / \alpha}} \mathrm{d} t\right]^{-\alpha /(\pi+\alpha)} \leq \lambda_{1}(T)
$$

Notice that this bound involves only the area and the smallest angle of a triangle.

Similarly we get a bound using [5], Theorem 6.3.

Theorem 2.5. Let $\beta=2 \arctan (h / d)$. We have

$$
j_{\frac{\pi}{\pi-\beta}}^{2} \frac{(\pi-\beta)^{2}}{d^{2} \cos ^{2}(\beta / 2)} f(\pi-\beta) \leq \lambda_{1}(T),
$$

where

$$
f(\gamma)=\left(4 \int_{0}^{1 / 2}\left[\frac{\gamma}{\sin (\gamma(1-t))}\right]^{2(1+\pi / \gamma)} \sin ^{2}(\pi t) \mathrm{d} t\right)^{-\gamma /(\gamma+\pi)}
$$

The last lower bound is an application of the Ricatti root method used in [7], Section 3.

Theorem 2.6. Let

$$
P(x, p)=-\frac{1+2 p}{2 x}+\frac{J_{1+p}(x)}{J_{p}(x)} .
$$


Then the smallest solution in $\lambda$ of the equation

$$
P\left(\sqrt{\lambda} a \cos \beta, \sqrt{\frac{1}{4}+\frac{\pi^{2}}{\tan ^{2} \beta}}\right)+P\left(\sqrt{\lambda} b \cos \alpha, \sqrt{\frac{1}{4}+\frac{\pi^{2}}{\tan ^{2} \alpha}}\right)=0
$$

yields a lower bound for $\lambda_{1}(T)$.

It is also possible to get new upper bounds by combining continuous symmetrization with [5], Theorem 2 .

$$
\lambda_{1}(T) \leq \inf _{h / d \leq \sin \beta \leq \sin \alpha} U(\beta),
$$

where

$$
U(\beta)=\left[\left(j_{\pi / \beta}^{2}-\frac{\pi^{2}}{\beta^{2}}\right) \int_{0}^{1}\left[\frac{g^{\prime}(\beta t)}{g(\beta t)}\right]^{2} \sin ^{2}(\pi t) \mathrm{d} t+\frac{j_{\pi / \beta}^{2}}{2}\right]\left[\int_{0}^{1} \frac{\sin ^{2}(\pi t)}{g^{2}(\beta t)}\right]^{-1}
$$

and

$$
g(\theta)=\frac{\sin (\beta-\theta)}{d \sin \beta}+\frac{\sin \theta}{h}
$$

This bound is quite complicated computationally. Thus, one may consider simplified bounds

$$
\begin{gathered}
\lambda_{1}(T) \leq U(\alpha) \quad([5], \text { Thm. 2), } \\
\lambda_{1}(T) \leq U(\arcsin (h / d)) \quad(\text { isosceles triangle). }
\end{gathered}
$$

The first is better for triangles close to subequilateral triangles, the second for those which are close to superequilateral triangles or almost degenerate cases.

Similarly we can use the upper bound from [5], Theorem 5.1.

Theorem 2.7. For arbitrary triangles we have

$$
\begin{aligned}
\lambda_{1}(T) \leq & \frac{\left(j_{\pi / \alpha}^{\prime}\right)^{2}}{A} \tan \alpha\left\{1-\left(\frac{\pi}{\alpha j_{\pi / \alpha}^{\prime}}\right)^{2}\left[1-\frac{1}{2 \int_{0}^{1} \sin ^{2}(\pi t) \sec ^{2}(\alpha t)}\right]\right. \\
& \left.-\frac{\pi / \alpha}{\left(j_{\pi / \alpha}^{\prime}\right)^{2}-(\pi / \alpha)^{2}} \frac{\int_{0}^{1} \tan (\alpha t) \sin (2 \pi t)}{\int_{0}^{1} \sin ^{2}(\pi t) \sec ^{2}(\alpha t)}\right\} .
\end{aligned}
$$

As in the previous section, we want to compare the bounds for various triangles. Figures 7-10 show the improvements that the functional bounds provide over the explicit bounds. Notice however, that Pólya's isoperimetric bound is still the best near the equilateral triangle. These figures show only the bounds that provide any improvement. The curves representing explicit bounds are also included, but are not labeled and are in gray. These are exactly the same curves as in the previous section. We omitted the plots of Conjecture 2.3 so as not to clutter the figures too much.

\subsection{Proofs}

In this section we prove the bounds for triangles. The proofs are quite short, as they follow directly from the combination of previous results and techniques. 


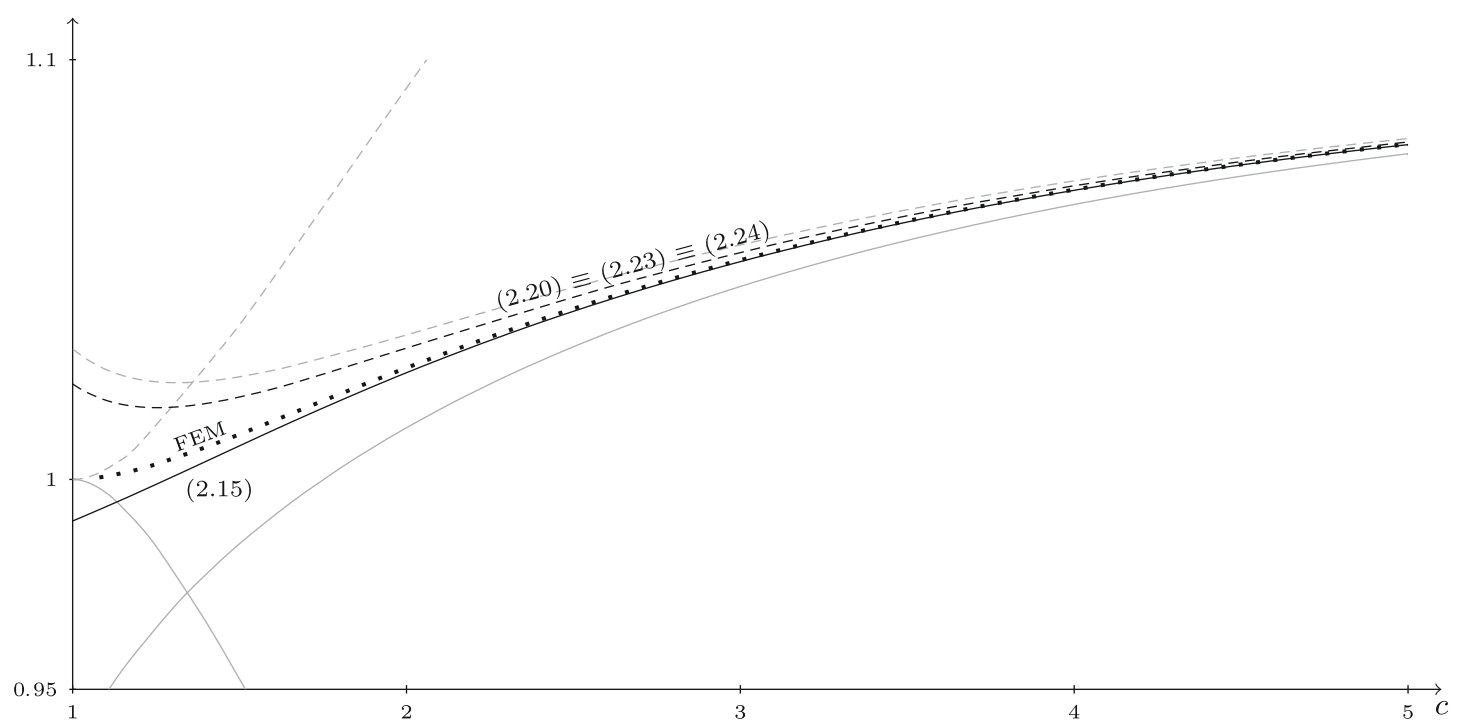

Figure 7. Comparison of functional lower bounds (solid curves) and functional upper bounds (dashed curves) for subequilateral triangles (with side lengths $1=a \leq b=c$ ). Each curve represents the ratio of the indicated bound over the conjectured lower bound (2.11) (Conjecture 2.3). The thick dotted line indicates numerical results (FEM with $2^{20}$ triangles). Faded curves show the explicit bounds from Figure 3.

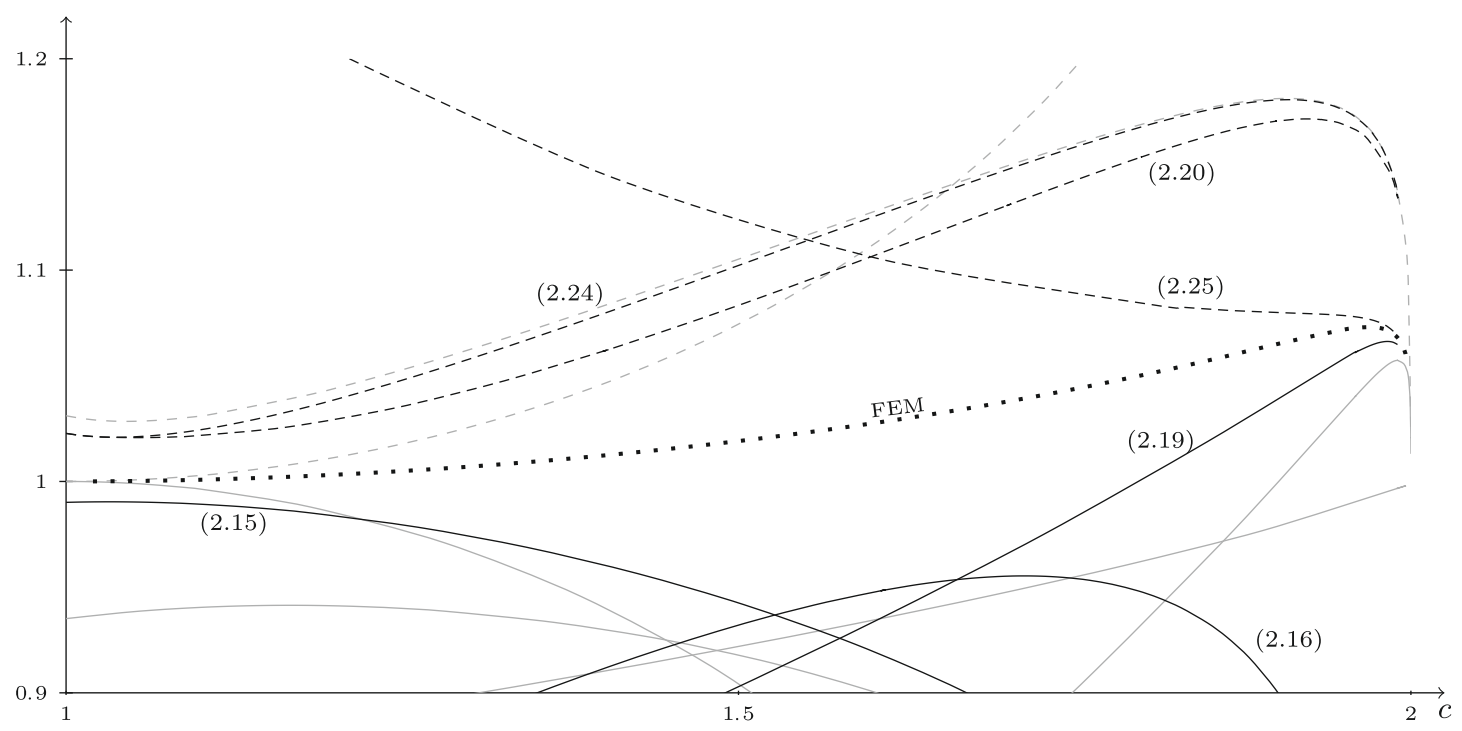

Figure 8. Comparison of functional lower bounds (solid curves) and functional upper bounds (dashed curves) for superequilateral triangles (with side lengths $1=a=b \leq c$ ). Each curve represents the ratio of the indicated bound over the conjectured lower bound (2.11) (Conjecture 2.3). The thick dotted line indicates numerical results (FEM with $2^{20}$ triangles). Faded curves show the explicit bounds from Figure 4. 


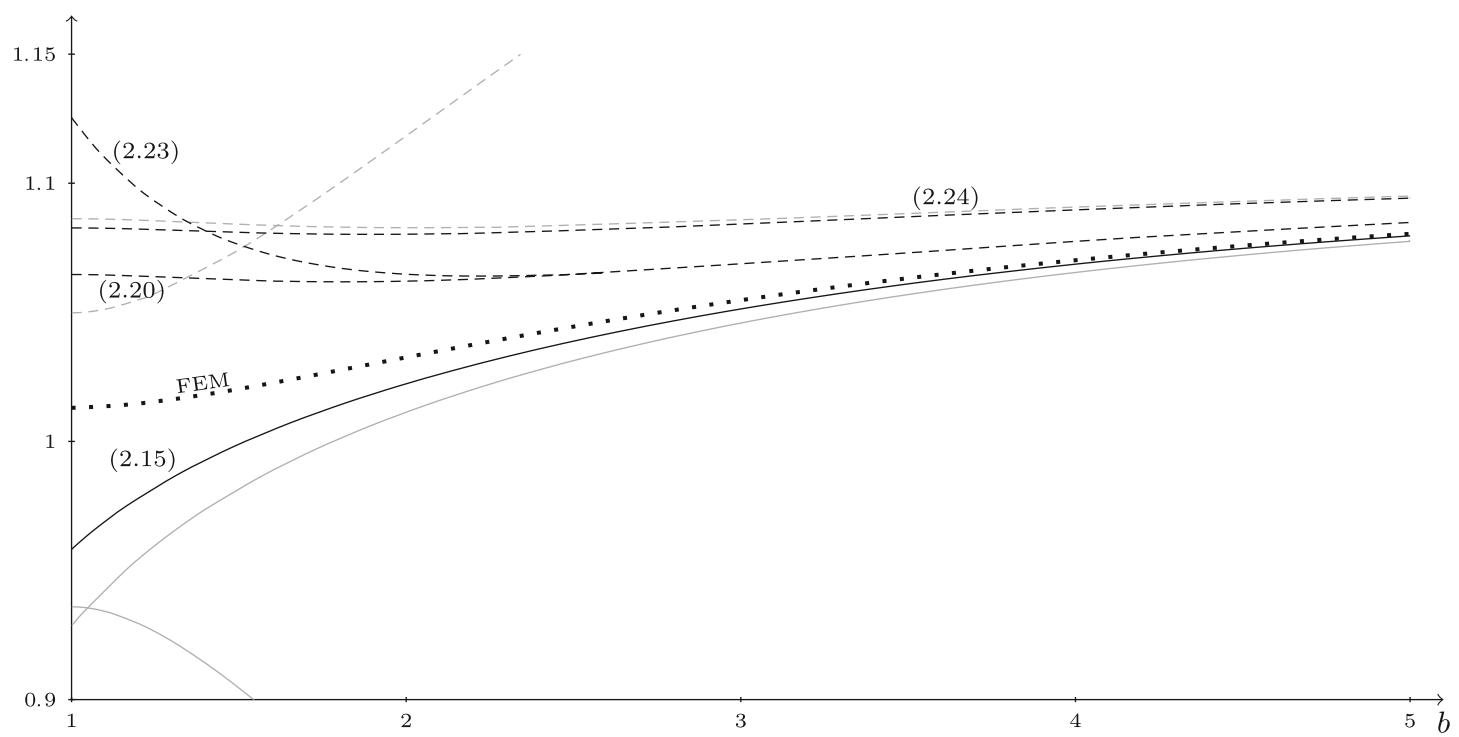

Figure 9. Comparison of functional lower bounds (solid curves) and functional upper bounds (dashed curves) for right triangles (with side lengths $1=a \leq b$ and $c=\sqrt{1+b^{2}}$ ). Each curve represents the ratio of the indicated bound over the conjectured lower bound (2.11) (Conjecture 2.3). The thick dotted line indicates numerical results (FEM with $2^{20}$ triangles). Faded curves show the explicit bounds from Figure 5.

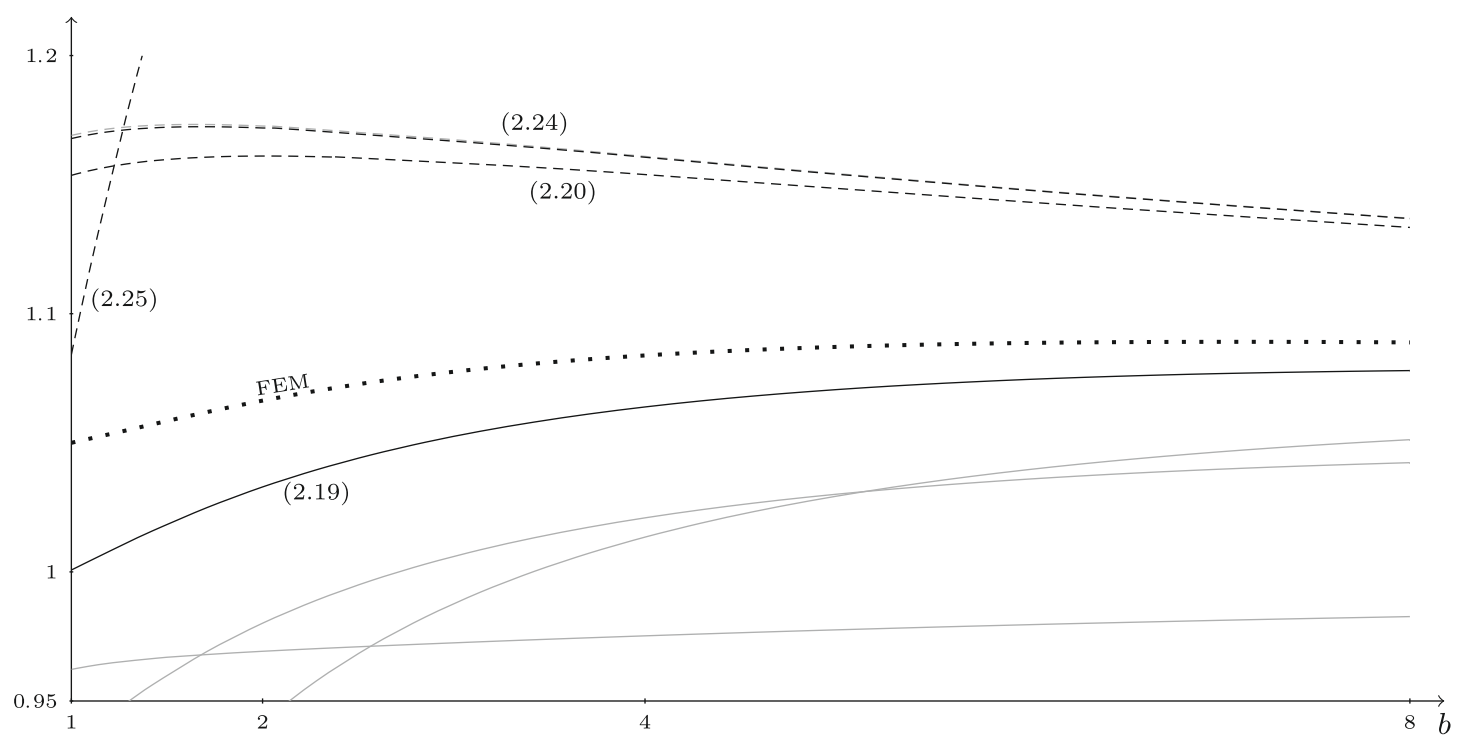

Figure 10. Comparison of functional lower bounds (solid curves) and functional upper bounds (dashed curves) for some nonisosceles triangles (with side lengths $1=a \leq b$ and $c=b+0.8$ ). Each curve represents the ratio of the indicated bound over the conjectured lower bound (2.11) (Conjecture 2.3). The thick dotted line indicates numerical results (FEM with $2^{20}$ triangles). Faded curves show the explicit bounds from Figure 6. 
Proof of Theorem 2.1. Let $T$ be an arbitrary triangle. We apply the continuous Steiner symmetrization to the isosceles triangle $T^{\prime}$ with the same diameter and the same shortest altitude as $T$. At a certain point we obtain $T$ as a symmetrized image of $T^{\prime}$. This gives an upper bound for the first eigenvalue of $T$. Now we take the largest circular sector contained in $T^{\prime}$. The radius of this sector equals the longest altitude of $T^{\prime}$ and the angle equals the smallest angle of $T^{\prime}$. This gives the desired result.

Proof of Theorem 2.2. We begin with the Steiner symmetrization of a triangle $T$ with respect to the line perpendicular to the longest side. This yields the isosceles triangle $T^{\prime}$ (usually superequilateral). If we apply [5], Proposition 2.4 , to $T^{\prime}$ we get (2.9). We can again symmetrize $T^{\prime}$ with respect to the longest side to get the rhombus. The proof follows using [5], Proposition 2.4.

The functional bounds from the previous section can be obtained in a similar fashion.

Proof of Theorem 2.4. This time we need [14], Lemma 4.1, to symmetrize a triangle into isosceles triangle with the same area and the same smallest angle. The result now follows from [5], Theorem 2.

Proof of Theorem 2.5. We proceed as in the proof of Theorem 2.2, except that we apply [5], Theorem 6.3, instead of Proposition 2.4 from the same paper.

Proof of Theorem 2.6. The proof is exactly the same as the proof of the case $\theta=0$ in [7], Section 3. We place a triangle in the coordinate system so that the longest side is contained in the $x$-axis. The only change needed is to adjust the heights of the cross-sections perpendicular to the $x$-axis.

Proof of (2.20). This bound is obtained in the same fashion as the bound in Theorem 2.1. The difference is that the optimal value for [5], Theorem 2, is not obtained at $T^{\prime}$, but at some triangle in between $T$ and $T^{\prime}$. Therefore numerical minimization in this range is needed. For simplicity, we can consider just the values at the endpoints $\left(T\right.$ and $\left.T^{\prime}\right)$. Also, for subequilateral triangles there is no optimization needed and the bound is equivalent to [5], Theorem 2.

Proof of Theorem 2.7. Given a superequilateral triangle we can apply [14], Lemma 4.1, to get any triangle with the same area, the same smallest angle and shorter shortest side. This new triangle will also have smaller first eigenvalue. This gives the upper bound for an arbitrary triangle if we apply [5], Theorem 5.1 to the superequilateral triangle whose equal angles are equal to the smallest angle of the triangle.

\section{BOUNDS FOR RHOMBI}

Here we present the best known bounds for eigenvalues of rhombi along with two new upper bounds. Throughout this section we assume the rhombus has vertices $(-d / 2,0),(0, a d / 2),(d / 2,0)$ and $(0,-a d / 2)$ where $0<a \leq 1$. This means that the longer diagonal has length $d$, shortest diagonal $a d$ and the opening angle has measure $\alpha=2 \arctan (a)$, and thus $a$ is the ratio between the lengths of the diagonals.

\subsection{Explicit bounds}

There are two bounds obtained by Hooker and Protter in [7] that will serve as base for comparisons, namely,

$$
\frac{\pi^{2}(1+a)^{2}}{a^{2} d^{2}} \leq \lambda_{1}(\mathcal{R}) \leq \frac{2 \pi^{2}\left(1+a^{2}\right)}{a^{2} d^{2}} .
$$

The lower bound is the best known for rhombi close to a square. There is a simple bound that is better than the above for small values of $a$, and which was obtained by the first author in [5], namely,

$$
\frac{4\left(j_{\pi / \alpha}^{\prime}\right)^{2} \cos ^{2}(\alpha / 2)}{d^{2}} \leq \lambda_{1}(\mathcal{R})
$$


In addition, there are two good upper bounds. The first was obtained by Pólya and Szegö:

$$
\lambda_{1}(\mathcal{R}) \leq \frac{\pi j_{0}^{2} \Gamma^{2}\left(\frac{\pi-\alpha}{2 \pi}\right)}{4 a^{2} d^{2} \Gamma^{2}\left(\frac{2 \pi-\alpha}{2 \pi}\right)} .
$$

The second is a bound good for thin rhombi obtained by including a rectangle:

$$
\lambda_{1}(\mathcal{R}) \leq \frac{\pi^{2}\left(1+a^{2 / 3}\right)^{3}}{a^{2} d^{2}} .
$$

Our first new bound is of the same nature as the lower bound given by (3.2).

Theorem 3.1. Let $\beta=\arcsin (a)$. Then

$$
\lambda_{1}(\mathcal{R}) \leq \frac{j_{\pi / \beta}^{2}}{d^{2} \cos ^{2}(\beta / 2)}
$$

The second result is a mixture of the bounds in (3.1). Notice that

$$
\frac{\pi^{2}(1+a)^{2}}{a^{2}}=\frac{2 \pi^{2}\left(1+a^{2}\right)}{a^{2}}-\frac{\pi^{2}(1-a)^{2}}{a^{2}}
$$

Hence the upper and lower bounds given by (3.1) differ by a second order term describing how far we are from a square. We have

\section{Theorem 3.2.}

$$
\lambda_{1}(\mathcal{R}) \leq \frac{2 \pi^{2}\left(1+a^{2}\right)}{a^{2} d^{2}}-0.796 \frac{\pi^{2}(1-a)^{2}}{a^{2} d^{2}} .
$$

This result shortens the gap between the upper and lower bounds of (3.1) by almost $80 \%$.

Figure 11 shows a comparison of all bounds from this section.

\subsection{Functional bounds}

In addition to the bounds from the previous section, it is possible to derive bounds involving either numerical integration or minimization. We also include these here since they provide better results than other bounds. For convenience we assume that the diameter $d$ of the rhombus has length 2 - to get general bounds one needs only to divide each bound by $(d / 2)^{2}$.

The first lower bound was obtained by Hooker and Protter in [7]:

$$
\min _{\lambda>0}\left\{J\left(\sqrt{\frac{1}{4}+\left(\frac{\pi}{2 a}\right)^{2}}, \sqrt{\lambda}\right)=-2 \sqrt{\lambda} J^{\prime}\left(\sqrt{\frac{1}{4}+\left(\frac{\pi}{2 a}\right)^{2}}, \sqrt{\lambda}\right)\right\} \leq \lambda_{1}(\mathcal{R}) .
$$

Another lower bound was obtained by the first author in [5].

$$
j_{\frac{\pi}{\pi-\alpha}}^{2} \frac{(\pi-\alpha)^{2}}{4 \sin ^{2}(\alpha / 2)} f(\pi-\alpha) \leq \lambda_{1}(\mathcal{R}),
$$

where

$$
f(\gamma)=\left(4 \int_{0}^{1 / 2}\left[\frac{\gamma}{\sin (\gamma(1-t))}\right]^{2(1+\pi / \gamma)} \sin ^{2}(\pi t) \mathrm{d} t\right)^{-\gamma /(\gamma+\pi)}
$$




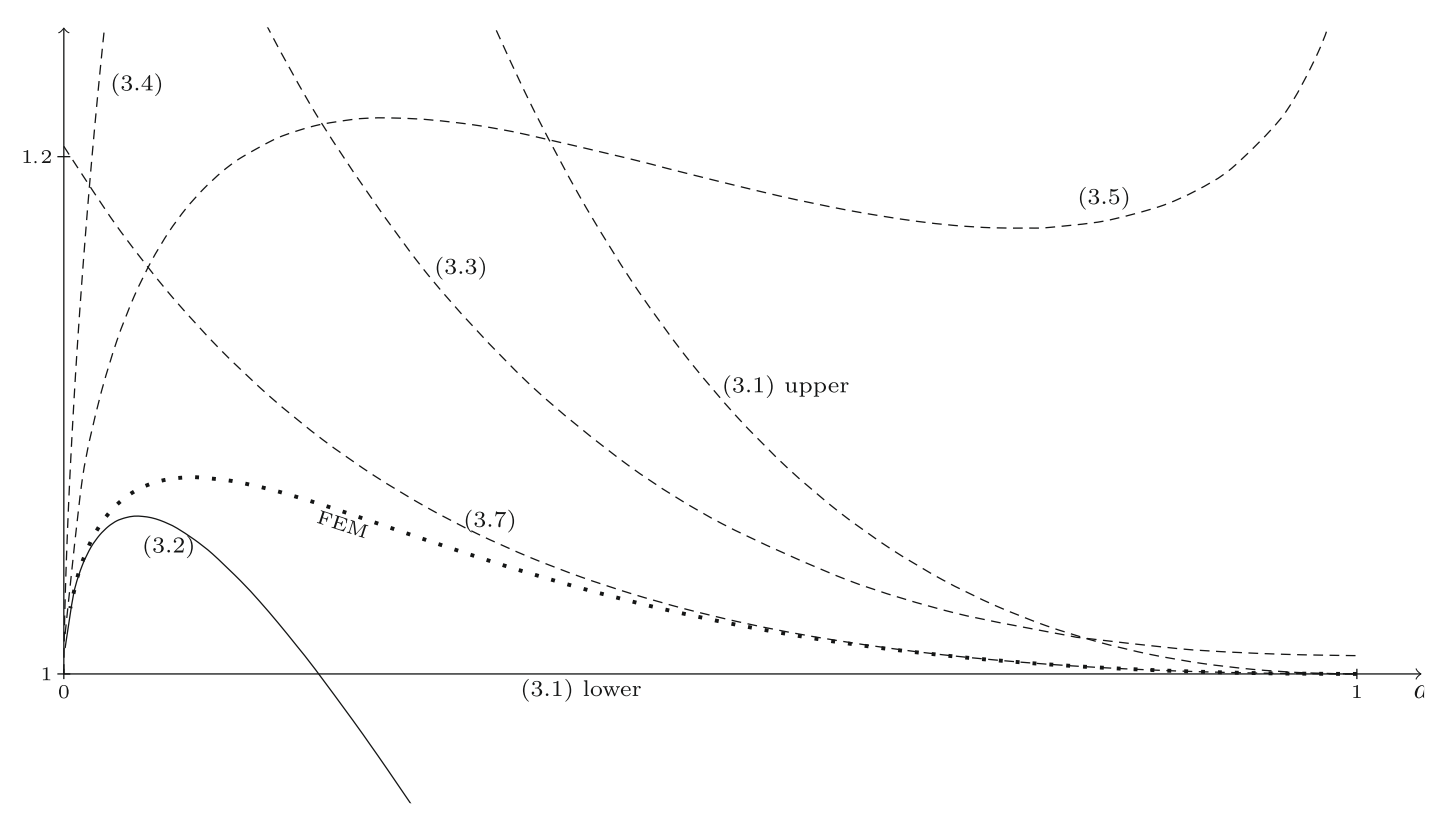

Figure 11. Comparison of lower bounds (solid curves) and upper bounds (dashed curves) for rhombi. Each curve represents the ratio of the indicated bound over the lower bound (3.1). The thick dotted line indicates numerical results (FEM with $2^{20}$ triangles).

We also have two new upper bounds similar to Theorems 3.1 and 3.2. The proofs are similar, but with numerically optimized parameters. The bound similar to that in Theorem 3.1 reads

$$
\lambda_{1}(\mathcal{R}) \leq \inf _{a / 2 \leq \tan (\beta / 2) \leq a} U(\beta)
$$

where

$$
U[\beta]=\left[\left(j_{\pi / \beta}^{2}-\frac{\pi^{2}}{\beta^{2}}\right) \int_{0}^{1 / 2}\left[\frac{g^{\prime}(\beta t)}{g(\beta t)}\right]^{2} \sin ^{2}(\pi t) \mathrm{d} t+\frac{j_{\pi / \beta}^{2}}{4}\right]\left[\int_{0}^{1 / 2} \frac{\sin ^{2}(\pi t)}{g^{2}(\beta t)}\right]^{-1}
$$

and

$$
g(\gamma)=\frac{\sin (\beta / 2-\gamma)}{a}+\frac{\sin \gamma}{2 \sin (\beta / 2)}
$$

The last upper bound is a variational bound similar to that in Theorem 3.2 but test functions are optimized for each specific rhombus. We first use a numerical minimization procedure to find the optimal coefficients in the linear combination and then use these coefficients to find a symbolic upper bound that is best for this particular rhombus. This means that there is no closed formula except for locally optimized bounds of the same form as the upper bound (3.1):

$$
\lambda_{1}(\mathcal{R}) \leq \frac{\pi^{2}\left(c_{1}+c_{2} a^{2}\right)}{2 a^{2}}
$$




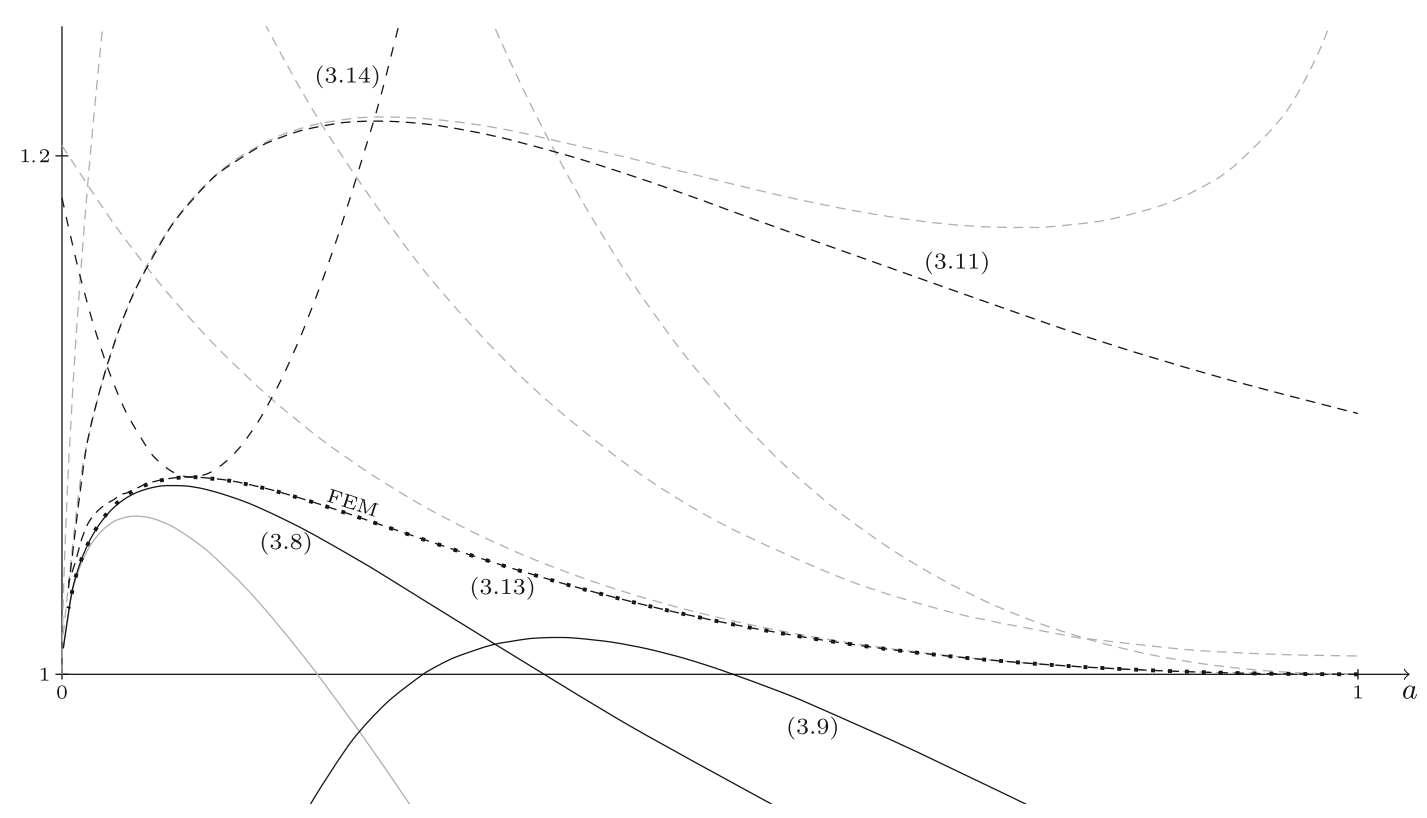

Figure 12. Comparison of functional lower bounds (solid curves) and functional upper bounds (dashed curves) for rhombi. Each curve represents the ratio of the indicated bound over the lower bound (3.1). The thick dotted line indicates numerical results (FEM with $2^{20}$ triangles). Faded curves show the explicit bounds from Figure 11.

TABLE 1. The difference (in percent) between FEM and variational bounds (3.7), (3.13).

\begin{tabular}{|c|c|c|c|c|}
\hline$a$ & 0.05 & 0.1 & 0.2 & 0.5 \\
\hline$\%(3.7)$ & 9.15456 & 5.62603 & 2.37409 & 0.15805 \\
\hline$\%(3.13)$ & 0.07817 & 0.01006 & 0.00557 & 0.00103 \\
\hline \hline$a$ & 0.8 & 0.85 & 0.9 & 0.95 \\
\hline$\%(3.7)$ & $2.7 \times 10^{-3}$ & $9.5 \times 10^{-4}$ & $2.6 \times 10^{-4}$ & $2.9 \times 10^{-5}$ \\
\hline$\%(3.13)$ & $2.92 \times 10^{-5}$ & $1.58 \times 10^{-6}$ & $-1.25 \times 10^{-5}$ & $-1.50 \times 10^{-5}$ \\
\hline
\end{tabular}

For example, the best bound around $a=0.1$ is

$$
\lambda_{1}(\mathcal{R}) \leq \frac{\pi^{2}\left(0.591913+5.914255 a^{2}\right)}{2 a^{2}}
$$

Figure 12 shows all bounds and a numerical values obtained using finite element method in MATLAB.

An interesting feature is that the variational upper bounds give almost the same results as a numerical method. It is even more surprising that FEM with $2^{22}$ triangles and 5 GB of memory usage gives higher values than the variational bound for $a \in(0.9,1)$ (Tab. 1 shows a comparison of FEM and the variational methods). This suggests that our variational bounds are quite close to the exact eigenvalues, and that, on the other hand, there is definitely room for improvement in the lower bounds.

One can also notice that there is no significant difference between the simple symmetrization bound (Thm. 3.1) and the bound (3.11). The difference is summarized in Table 2. 
TABLE 2. The difference (in percent) between (3.11) and (3.5).

\begin{tabular}{|c|c|c|c|c|c|c|c|}
\hline$a$ & 0.005 & 0.01 & 0.02 & 0.05 & 0.1 & 0.2 & 0.4 \\
\hline$\%$ & 0.00009 & 0.00037 & 0.00135 & 0.00701 & 0.02372 & 0.08652 & 0.42814 \\
\hline
\end{tabular}

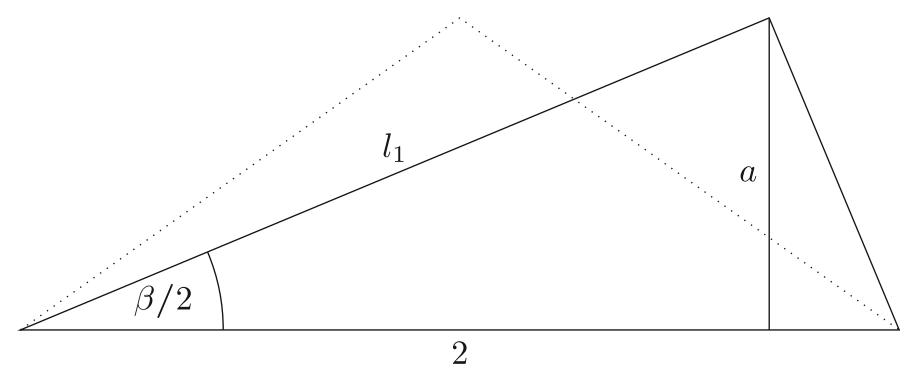

Figure 13. Partially dessymmetrized rhombus.

\subsection{Proofs}

In this section we prove all new results for rhombi. Without loss of generality we can assume that the length of the diameter $d$ equals 2. Let us begin with the proof of Theorem 3.1.

Proof of Theorem 3.1. Consider a rhombus with diagonals of length 2 and $2 a$. This implies that the angle opening $\alpha=2 \arctan (a)$. Consider an isosceles triangle with shortest side of length $2 a$ and altitude perpendicular to this side of length 2 . If we perform a continuous symmetrization of this triangle we get a family of quadrilaterals with perpendicular diagonals of the same length as the diagonals of the rhombus. In particular there exists exactly one quadrilateral in this family which has two right angles and whose smallest eigenvalue is larger than that of the rhombus. Let $\beta$ be the smallest angle in this quadrilateral. Then (see Fig. 13)

$$
\begin{aligned}
& \sin \frac{\beta}{2}=\frac{a}{l_{1}}, \\
& \cos \frac{\beta}{2}=\frac{l_{1}}{2} .
\end{aligned}
$$

These two equations give

$$
\sin \beta=a,
$$

also

$$
l_{1}=2 \cos \frac{\beta}{2} .
$$

Inside of this quadrilateral we can put a circular sector with angle $\beta$ and radius $l_{1}$. Its eigenvalue gives the desired upper bound.

We could also use different intermediate steps of continuous symmetrization of the isosceles triangle, or even the isosceles triangle itself. Another natural choice would be a point when $l_{1}=2$ (quadrilateral splits into two isosceles triangles). Table 3 shows upper bounds obtained from the mentioned steps of symmetrization.

Theorem 2.1 is good only for thin rhombi. The table shows that it is best to include a sector the way it is done in the theorem.

In general we could pick $\beta$ which gives the optimal result (a quadrilateral somewhere between two right triangles and two isosceles triangles), but this has to be done numerically. In such a case we may as well use 
TABLE 3. The difference between upper bounds obtained by including the largest possible circular sector in different quadrilaterals (in \% compared to Thm. 2.1).

\begin{tabular}{|c|c|c|c|c|}
\hline$a$ & 0.05 & 0.1 & 0.2 & 0.3 \\
\hline Isosceles triangle & $0.05317 \%$ & $0.19244 \%$ & $0.64941 \%$ & $1.24597 \%$ \\
\hline Two isosceles triangles & $0.01096 \%$ & $0.03376 \%$ & $0.07510 \%$ & $0.06106 \%$ \\
\hline \hline$a$ & 0.4 & 0.5 & 0.7 & 0.9 \\
\hline Isosceles triangle & $1.87917 \%$ & $2.44359 \%$ & $2.80224 \%$ & $-0.04319 \%$ \\
\hline Two isosceles triangles & $-0.06310 \%$ & $-0.35912 \%$ & $-1.85243 \%$ & $-6.32744 \%$ \\
\hline
\end{tabular}

TABLE 4. The optimal sector compared to the optimal numerical integration.

\begin{tabular}{|c|c|c|c|c|}
\hline$a$ & 0.05 & 0.1 & 0.2 & 0.3 \\
\hline$\%$ & 0.09794 & 0.20819 & 0.40159 & 0.55155 \\
\hline \hline$a$ & 0.4 & 0.5 & 0.7 & 0.9 \\
\hline$\%$ & 0.66222 & 0.73919 & 0.81145 & 0.80214 \\
\hline
\end{tabular}

the upper bound proved by the first author in [5], which is more complicated than the included sector bound, but gives slightly better results. This gives the proof of (3.11). Table 4 shows a comparison between optimized sector and optimized numerical integration.

The next proof is an application of the variational method with transplanted eigenfunctions used in $[4,7,13,14]$.

Proof of Theorem 3.2. The idea of the proof is to transplant eigenfunctions of triangles into rhombi in as many ways as possible. There are two natural ways of stretching triangles to fit to rhombi. One is to divide a triangle with a line of symmetry into two identical triangles and to transplant each of them into halves of a rhombus. This would give a function which is positive inside of a rhombus, hence approximating the first eigenfunction. The other method is to stretch a whole triangle into a half of a rhombus. This approximates the second eigenfunctions (the second eigenfunction of a square is equal to the first eigenfunction of a right isosceles triangle).

One possible list of known eigenfunctions with different ways of transplanting them is included below.

Note that due to the symmetry of a rhombus we only need to transplant eigenfunctions into the whole rhombus, the left half and the upper half. The first case gives functions positive on the whole rhombus, hence similar to the first eigenfunction of the rhombus. Stretching onto halves gives test functions similar to the second eigenfunction. We can even restrict our considerations to only the upper left quarter and calculate the Rayleigh quotient on this domain. Such test functions are clearly continuous and still satisfy Dirichlet boundary condition on the whole rhombus.

Let $z=\frac{\pi}{3}(2 x-1)$ and $t=\pi\left(1-\frac{2}{\sqrt{3}} y\right)$. We have:

- The first eigenfunction of the square $[0,1] \times[0,1]$,

$$
\varphi_{1}(x, y)=\sin (\pi x) \sin (\pi y),
$$

- stretches linearly into a whole rhombus.

- The first eigenfunction of the right isosceles triangle with vertices at $(0,0),(0,1)$ and $(1,0)$,

$$
\varphi_{2}(x, y)=\sin (\pi x) \sin (2 \pi y)+\sin (2 \pi x) \sin (\pi y)
$$

- stretches linearly into upper or left half (3 ways each depending where the longest side is mapped);

- stretches into a whole rhombus (4 ways depending on which vertex of a rhombus is mapped to the midpoint of the longest side of a triangle). 
- The first eigenfunction of the equilateral triangle with vertices at $(0,0),(1,0)$ and $(1 / 2, \sqrt{3} / 2)$,

$$
\varphi_{3}(x, y)=2 \cos (3 z) \sin (t)-\sin (2 t)
$$

- stretches linearly into upper or left half;

- stretches into a whole rhombus (4 ways).

- The first eigenfunction of a right triangle with angle $\pi / 3$ with vertices at $(0,0),(1 / 2,0)$ and $(1 / 2, \sqrt{3} / 2)$,

$$
\varphi_{4}(x, y)=\sin (4 z) \sin (2 t)-\sin (5 z) \sin (t)-\sin (z) \sin (3 t)
$$

- stretches linearly into upper or left half (6 ways each);

- stretches into a whole rhombus $(8+8$ ways, due to lack of symmetry we can either split the longest side at the midpoint, or where the altitude cuts it).

This already gives 45 possible terms in a linear combination. Of course one could come up with even more which would certainly improve the final variational bound. It might be interesting, for example, to consider polynomial functions defined on quarters of a rhombus which are zero on a side and increasing toward the center. Adding more test functions is perhaps not very interesting from a theoretical point of view, but as Table 1 shows, this gives a resource-friendly method of obtaining upper bounds that are as good as the finite element method. An added advantage is that the bounds are symbolic with only rational numbers involved, hence there is no numerical error involved.

To prove Theorem 3.2 we "only" need 17 test functions. The optimized bound (3.14) ((3.13) in general) is based on all 45 test functions. We form a linear combination of: the first eigenfunction of a square; the first eigenfunction of a right isosceles triangle mapped ( 4 ways) into a whole rhombus and (2 ways) into halves with the longest side mapped into diagonals; the first eigenfunction of an equilateral triangle mapped (4 ways) into a whole rhombus and ( 2 ways) into halves; the first eigenfunction of a right triangle with angle $\pi / 3$ mapped (4 ways) into a whole rhombus with the midpoint of the longest side mapped to one of the vertices and the angle equal $\pi / 3$ in the right or upper half of a rhombus. These 17 transplantations are the most natural choices and they are needed to prove the result.

Since the calculations involved are extremely long, we use Mathematica to handle them. This is done by means of the script in Appendix 2. Our goal is to explain each step of the proof. We plug the linear combination into the Rayleigh quotient to get an upper bound depending on 17 unknowns $\left(\{c(i)\}_{i=0}^{16}\right)$. The integrals involved are very simple, as they are all of the form $\sin (A+B x+C y)$ or $\cos (A+B x+C y)$ integrated over the triangle with vertices $(0,0),(1,0)$ and $(0, a)$. There are, however, literally thousands of such integrals, this being the reason to use the script. This gives an upper bound for the triangle with Dirichlet boundary conditions on $(1,0)-(0, a)$ and Neumann boundary conditions on the other sides, which is equivalent to getting an upper bound for a rhombus with Dirichlet boundary conditions.

Now we can perform a numerical minimization to find coefficients for a wide range of rhombi. Due to the very high dimension of the space of coefficients and the nonlinear character of the bound we cannot expect to get a global minimum. Nevertheless, for a given value of $a$ we obtain certain values of coefficients which are then plugged back into the bound. This gives a symbolic bound optimized for this specific value of $a$, which is also an upper bound (although not optimal) for any other value $a$ (for example bound (3.14)).

To get the uniform upper bound (3.7) we need numerical coefficients for 1000 values of $a$ in the range from 0 to 1 . Having obtained these, we can use a least-square method to fit polynomials (in $a$ ) to the data for each coefficient. This is a numerical process, but we can rationalize the resulting polynomials to get a symbolic upper bound involving only $a$. Of course this bound is an extremely complicated rational function, thus we need to show that it is smaller than (3.7).

To get a well-fitted polynomial we need data that appear to be smooth. With 17 coefficients it is impossible to guarantee this using numerical minimization at each sample point. To make the data more consistent we start with a known optimal solution on a square. We then look for a local minimum satisfying a normalizing condition on the sum of the squares of the coefficients, say equal to one, and choose the starting point based 


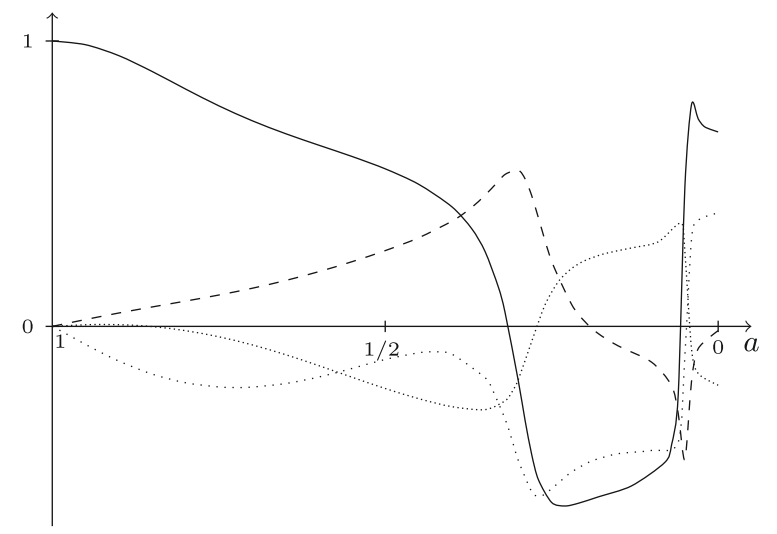

FIGURE 14. Plots of numerical data for some of the coefficients against the parameter $a$.

on previous results. That is, we consider $a(i)=1-i / 1000$ with $a(0)$ corresponding to a square which has a known solution (coefficients are 0 for every function except for the eigenfunction of to the square). The initial point for the next step is either the solution for the last step, or the extrapolated value of the last two steps (whichever gives a smaller minimum). This procedure gives smooth enough data. Figure 14 shows plots of the data for some of the constants. The graphs for all constants are drawn by the script.

Based on the data for values of $a$ between 0.35 and 0.1 we fit a degree 10 polynomial to all coefficients. To get a symbolic bound we also approximate coefficients of all polynomials by rational numbers with small denominators. While the script gives all those polynomials, here we just include two as examples:

$$
\begin{aligned}
c(0)= & -\frac{16265}{81}+\frac{75626}{25} a-\frac{551380}{27} a^{2}+\frac{2520297}{31} a^{3}-\frac{7596695}{36} a^{4} \\
+ & \frac{7828991}{21} a^{5}-\frac{17697419}{39} a^{6}+\frac{4131617}{11} a^{7}-\frac{9910213}{49} a^{8} \\
+ & \frac{2557973}{40} a^{9}-\frac{90131}{10} a^{10}, \\
c(1)= & \frac{4}{27}(1-a)-\frac{5}{27}(1-a)^{2}-\frac{441}{32}(1-a)^{3}+\frac{1025}{9}(1-a)^{4} \\
& -\frac{25035}{37}(1-a)^{5}+\frac{77172}{29}(1-a)^{6}-\frac{212347}{32}(1-a)^{7} \\
& +\frac{312154}{31}(1-a)^{8}-\frac{337629}{40}(1-a)^{9}+\frac{185247}{62}(1-a)^{10}
\end{aligned}
$$

Note that $c(0)$ is a polynomial in $a$, but $c(1)$ (and other constants) are polynomials in $1-a$ with no free term. This guarantees that the test function is equal to the eigenfunction of a square when $a=1$.

Finally, we use the algorithm from [14] to prove that the resulting rational function is smaller than (3.7). To do this we first rewrite the inequality in polynomial form and then show that inequality is true for all values of $a$ in the interval $(0.1,1)$.

Similarly, based on the data for the range 0.06 to 0.2 we fit a degree 5 polynomial getting an inequality holding for the interval $(0.06,0.1)$.

To finish the proof (for $a \leq 0.06$ ) we will use Theorem 3.1. We need an upper bound for the zeros of the Bessel function proved in [12]. We have

$$
j_{v} \leq v-\frac{a_{1}}{\sqrt[3]{2}} \sqrt[3]{v}+\frac{3 a_{1}^{2} \sqrt[3]{2}}{20 \sqrt[3]{v}}
$$


where $a_{1} \approx-2.3381$ is the first zero of the Airy function. This gives

$$
\begin{aligned}
\beta^{2} \lambda_{1} & \leq\left(1-\frac{a_{1}}{\sqrt[3]{2}}\left(\frac{\pi}{\beta}\right)^{-2 / 3}+\frac{3 a_{1}^{2} \sqrt[3]{2}}{20}\left(\frac{\pi}{\beta}\right)^{-4 / 3}\right)^{2} \frac{\pi^{2}}{4 \cos ^{2}(\beta / 2)} \\
& \leq\left(1-\frac{a_{1}}{\sqrt[3]{2}}\left(\frac{\pi}{\beta}\right)^{-2 / 3}+\frac{3 a_{1}^{2} \sqrt[3]{2}}{20}\left(\frac{\pi}{\beta}\right)^{-4 / 3}\right)^{2} \frac{\pi^{2}}{4-\beta^{2}}
\end{aligned}
$$

We want to show that

$$
\begin{aligned}
\beta^{2} \lambda_{1} & \leq \frac{\pi^{2} \beta^{2}}{2 a^{2}}\left(1+a^{2}-0.398(1-a)^{2}\right) \\
& =\frac{\pi^{2} \beta^{2}}{2}\left(0.602+\frac{0.796}{a}+\frac{0.602}{a^{2}}\right) \\
& =\frac{\pi^{2} \beta^{2}}{2}\left(0.602+\frac{0.796}{\arcsin \beta}+\frac{0.602}{\arcsin ^{2} \beta}\right) \\
& \leq \frac{\pi^{2}}{2}\left(0.602 \beta^{2}+0.796 \beta+0.602\right) .
\end{aligned}
$$

Therefore we need

$$
2\left(1-\frac{a_{1}}{\sqrt[3]{2}}\left(\frac{\beta}{\pi}\right)^{\frac{2}{3}}+\frac{3 a_{1}^{2} \sqrt[3]{2}}{20}\left(\frac{\beta}{\pi}\right)^{\frac{4}{3}}\right)^{2} \leq\left(0.602 \beta^{2}+0.796 \beta+0.602\right)\left(4-\beta^{2}\right) .
$$

Substituting $a_{1} \rightarrow-2.34$ increases the left side while writing $\gamma^{3}=\beta$ changes this inequality into a polynomial inequality. We can use the same algorithm as in the other cases to show that this inequality holds for $\gamma<0.395$ (equivalent to $a<0.06$ ).

This completes the proof of Theorem 3.2 .

\section{BOUndS FOR QUADRILATERALS}

We first prove the lower bound in Theorem 1.1, which is based on Hooker and Protter's lower bound for the rhombus given by (3.1).

Proof of the lower bound from Theorem 1.1. Take as the axis in the Steiner symmetrization a line perpendicular to the longest diagonal of the quadrilateral $Q$ which is contained in $Q$. With the notation in the theorem, this gives a new quadrilateral $Q^{\prime}$ with two perpendicular diagonals of lengths $d_{1}^{\prime}=d_{1} \sin \theta$ and $d_{2}$. Symmetrizing again, now with respect to the line containing the diagonal of length $d_{2}$, we obtain a rhombus $(\mathcal{R})$ with side length $\ell$ and opening angle $\alpha$ satisfying

$$
\sin \left(\frac{\alpha}{2}\right)=\frac{d_{2}}{2 \ell} \quad \text { and } \quad \cos \left(\frac{\alpha}{2}\right)=\frac{d_{1} \sin \theta}{2 \ell} .
$$

This yields

$$
\sin \alpha=\frac{d_{1} d_{2} \sin \theta}{2 \ell^{2}} \quad \text { and } \quad \ell^{2}=\frac{1}{4}\left(d_{1}^{2} \sin ^{2} \theta+d_{2}^{2}\right) .
$$

Since, from the symmetrization, we have $\lambda_{1}(Q) \geq \lambda_{1}\left(Q^{\prime}\right) \geq \lambda_{1}(\mathcal{R})$, the lower bound (3.1) gives, after some rewriting, the desired result. 
Corollary 4.1. Let $T$ be a triangle with diameter $d$ and height with respect to the side of length $d$ denoted by $h$. Then

$$
\pi^{2} \frac{(d+h)^{2}}{d^{2} h^{2}}=\frac{\pi^{2}}{4 A^{2}}\left(d+\frac{2 A}{d}\right)^{2} \leq \lambda_{1}(T) .
$$

Proof. Apply the lower bound in Theorem 1.1 to $T$ taking as the fourth vertex any point on the side of length $d$.

As mentioned in the Introduction, the method used in the proof of the lower bound in Theorem 1.1 may be applied to other lower bounds for the rhombus to produce lower bounds for quadrilaterals. If we apply it to the lower bound obtained in [5] instead (see (3.2)) we get

Theorem 4.2. Under the same conditions and notation as in Theorem 1.1 we have

$$
\frac{4\left(j_{\frac{\pi}{\alpha}}^{\prime}\right)^{2}}{d_{1}^{2} \sin ^{2} \theta+d_{2}^{2}} \leq \lambda_{1}(Q)
$$

where $\alpha$ satisfies

$$
\sin \alpha=\frac{2 d_{1} d_{2} \sin \theta}{d_{1}^{2} \sin ^{2} \theta+d_{2}^{2}} .
$$

We now prove the second part of Theorem 1.1.

Proof of the upper bound from Theorem 1.1. This proof is an extension of the proof of the upper bound (3.1) proved in [7]. There, the first eigenfunction of a square is linearly transformed into a function on a rhombus. Next, this function is used as a test function in the Rayleigh quotient to obtain an upper bound. This method was also used for triangles in [4] and later in [13,14]. In these last two cases the test function is a linear combination obtained from eigenfunctions for different triangles. We intend to use this method here. An arbitrary quadrilateral (up to scaling) can be represented on a plane by four vertices: $(-1,0),(c,-d),(1,0)$ and $(a, b)$. We can assume that $b>d>0$ so that the diagonal inside of a quadrilateral is contained in the $x$-axis. The area of such quadrilateral is equal to $b+d$. We can also assume that $a^{2}+b^{2} \geq 1$ (upper triangle is acute) since there is always at least one acute angle in a quadrilateral.

Unfortunately, there is essentially only one quadrilateral with known eigenfunctions, namely the square. All eigenfunctions of rectangles come from linearly transformed eigenfunctions of the square. Hence we need to use eigenfunctions of triangles to get a better result than (3.1) (or the second eigenfunction of a square). The second eigenfunction of a square with a diagonal nodal line is a first eigenfunction of a right isosceles triangle. Let $\varphi(x, y)$ be the first eigenfunction of the square with vertices $(0,0),(1,0),(1,1)$ and $(0,1)$ and $\psi(x, y)$ be the first eigenfunction of the right isosceles triangle with vertices $(0,0),(1,0)$ and $(0,1)$. Let also $L_{1}$ be a linear transformation that moves points $(0,0),(1,0)$ and $(0,1)$ into points $(-1,0),(1,0)$ and $(a, b)$ (upper half of a quadrilateral). Finally let $L_{2}$ transform the same three points into $(-1,0),(1,0)$ and $(c,-d)$ (lower half of a quadrilateral). We could use the first two eigenfunctions of a square to get the following test function for the Rayleigh quotient:

$$
\begin{gathered}
f(x, y)=\varphi(x, y)+\alpha\left(\left(\psi \circ L_{1}\right)(x, y)-\left(\psi \circ L_{2}\right)(x, y)\right), \\
\varphi(x, y)=\sin (\pi x) \sin (\pi y) \\
\psi(x, y)=\sin (\pi x) \sin (2 \pi y)+\sin (2 \pi x) \sin (\pi y) .
\end{gathered}
$$

Notice that the second eigenfunction of a square is composed of the first eigenfunctions of a right triangle and is antisymmetric with respect to diagonal. Instead of the above we can get a better result if we use

$$
f(x, y)=\varphi(x, y)+\alpha\left(\psi \circ L_{1}\right)(x, y)-\beta\left(\psi \circ L_{2}\right)(x, y)
$$


The difference is that we can change the balance between the upper and lower part depending on the area of each part ( $d$ compared to $b)$. This leads to a bound

$$
\begin{aligned}
\lambda_{1}(Q) \leq & \frac{\left(1+a^{2}+b^{2}\right)\left(45 \pi^{2}\left(1+5 \alpha^{2}\right)-128 \alpha(-11+5 \alpha)\right)-128 \alpha(6-10 \alpha)}{2 b\left(b\left(45+(1024 \alpha) / \pi^{2}+90 \alpha^{2}\right)+d\left(45-(1024 \beta) / \pi^{2}+90 \beta^{2}\right)\right)} \\
& +\frac{\left(1+c^{2}+d^{2}\right)\left(45 \pi^{2}\left(1+5 \beta^{2}\right)-128 \beta(11+5 \beta)\right)+128 \beta(6+10 \beta)}{2 d\left(b\left(45+(1024 \alpha) / \pi^{2}+90 \alpha^{2}\right)+d\left(45-(1024 \beta) / \pi^{2}+90 \beta^{2}\right)\right)} .
\end{aligned}
$$

We need to show that this quantity is smaller than the bound in the theorem. Before we proceed, we want to change variables to get a simpler statement. Notice that

$$
\begin{aligned}
& l_{1}^{2}+l_{2}^{2}=(a+1)^{2}+b^{2}+(a-1)^{2}+b^{2}=2\left(a^{2}+b^{2}+1\right), \\
& l_{3}^{2}+l_{4}^{2}=(c+1)^{2}+d^{2}+(c-1)^{2}+d^{2}=2\left(c^{2}+d^{2}+1\right) .
\end{aligned}
$$

Put

$$
\begin{gathered}
x=a^{2}+b^{2}+1 \geq 2, \\
y=c^{2}+d^{2}+1 \geq 1, \\
0<z=d / b \geq 1 .
\end{gathered}
$$

After this change of variables the bound from the theorem reads

$$
\lambda_{1}(Q) \leq \frac{\pi^{2}}{b^{2}} \frac{x+y}{(1+z)^{2}},
$$

and the variational bound (4.5) changes into

$$
\begin{aligned}
\lambda_{1}(Q) \leq & \frac{\pi^{2}}{b^{2}}\left[\frac{45 \pi^{2}\left(x z\left(1+5 \alpha^{2}\right)+y\left(1+5 \beta^{2}\right)\right)}{2 z\left(1024(\alpha-z \beta)+45 \pi^{2}\left(1+z+2 \alpha^{2}+2 z \beta^{2}\right)\right)}\right. \\
& \left.-\frac{128(z \alpha(6-11 x-10 \alpha+5 x \alpha)+\beta(-6+11 y-10 \beta+5 y \beta))}{2 z\left(1024(\alpha-z \beta)+45 \pi^{2}\left(1+z+2 \alpha^{2}+2 z \beta^{2}\right)\right)}\right] .
\end{aligned}
$$

Notice that the ratio of (4.13) and (4.12) depends only on three variables $x, y$ and $z$. Our goal is to show that for any $x \geq 2, y \geq 1$ and $0<z \leq 1$ there exist $\alpha$ and $\beta$ such that the ratio is not greater than 1 . First notice that the denominator of (4.13) is always positive since it is equal to the second norm of $f(x, y)$. This implies that the denominator of the ratio is also positive. Therefore it is enough to show that the numerator of the ratio is smaller than the denominator. This gives a polynomial inequality with three variables and two parameters:

$$
\begin{aligned}
0 \geq P(x, y, z ; \alpha, \beta)= & -45 \pi^{2}(y-x z)\left(z^{2}-1\right) \\
& +128 z\left(-16(x+y)+5(1+z)^{2}+11(x-1)(1+z)^{2}\right) \alpha \\
& +5 z\left(-36 \pi^{2}(x+y)-128(x-2)(1+z)^{2}+45 \pi^{2} x(1+z)^{2}\right) \alpha^{2} \\
& +128\left(16(x+y) z^{2}-5(1+z)^{2}-11(y-1)(1+z)^{2}\right) \beta \\
& +5\left(-128(y-2)(1+z)^{2}+9 \pi^{2}\left(-4 x z^{2}+y(z+5)^{2}\right)\right) \beta^{2} .
\end{aligned}
$$

We begin with quadrilaterals for which $z$ is small. This means that the area of the upper triangle is much larger than the area of the lower triangle. Therefore the quadrilateral looks almost like a triangle. This implies 
that $\alpha$ must be large to diminish the influence of the first eigenfunction of the square and that $\beta$ should be small. We take $\beta=0$ and $\alpha=1 /(7 z)$. The constant 7 has been chosen by trial and error. This particular choice ensures that near the degenerate case $z=0$ the test function is almost exclusively based on an upper triangle. We get

$$
\begin{aligned}
49 z P(x, y, z ; 1 /(7 z), 0)= & 128\left(5-179 z+75 z^{2}+35 z^{3}\right) \\
& +45 \pi^{2}\left(-3+59 z-44 z^{2}-49 z^{3}+49 z^{4}\right) \\
& +y\left(-14336 z-45 \pi^{2}\left(4-49 z+49 z^{3}\right)\right) \\
& +x\left(128\left(-5-45 z+149 z^{2}+77 z^{3}\right)\right. \\
& \left.+45 \pi^{2}\left(1+10 z-44 z^{2}+49 z^{4}\right)\right) .
\end{aligned}
$$

This is the first order polynomial in $x$ and $y$ with parameter $z$. Each coefficient is a polynomial in $z$ of order at most 4 . First we substitute $x=x^{\prime}+2$ and $y=y^{\prime}+1$ so that $x^{\prime} \geq 0$ and $y^{\prime} \geq 0$. Now we find the roots for all the coefficients. We get only two real roots satisfying $0 \leq z \leq 1(z \approx 0.344$ and $z \approx 0.565)$. By checking the value of each coefficient at $z=0.25$ we get that all coefficients are negative for $0 \leq z \leq 1 / 3$. This proves the theorem for such $z$.

The case $z=1$ is trivial, since $P(x, y, 1 ; 0,0)=0$.

We are left with $x \geq 2, y \geq 1$ and $1 / 3<z<1$. If $z$ is close to 1 the upper and lower triangles have almost the same area. Therefore we need a similar contribution from $\alpha$ and $\beta$. Taking into account the previous case $(\beta=0$ for small $z)$ it is reasonable to try $\beta=\alpha z$. Since $P(x, y, z ; \alpha, \alpha z)$ is a quadratic equation in $\alpha$, we will be done if we can show that the discriminant is nonnegative. Denoting it by $D(x, y ; z)$, we have that $D(x, y ; z)$ is a quadratic polynomial in $x, y$ and a higher order polynomial in $z$. We can thus treat it as the equation of a conic with parameter $z$.

Let

$$
\begin{gathered}
\Delta=\left|\begin{array}{lll}
a & b & d \\
b & c & f \\
d & f & g
\end{array}\right|, \\
J=\left|\begin{array}{ll}
a & b \\
b & c
\end{array}\right|, \\
I=a+c .
\end{gathered}
$$

A conic given by the equation below is an ellipse if $J>0$ and $I \Delta<0$ :

$$
a x^{2}+2 b x y+c y^{2}+2 d x+2 f y+g=0 .
$$

An ellipse with such equation is the same as the ellipse

$$
\frac{\left(x-x_{0}\right)^{2}}{a_{0}^{2}}+\frac{\left(y-y_{0}\right)^{2}}{b_{0}^{2}}=1,
$$

rotated by the angle $\operatorname{arccot}((a-c) / 2 b) / 2$. The center and semiaxes of this ellipse satisfy

$$
\begin{gathered}
x_{0}=\frac{b f-c d}{J}, \\
y_{0}=\frac{b d-a f}{J}, \\
a_{0}^{2}+b_{0}^{2}=-\frac{I \Delta}{J^{2}} .
\end{gathered}
$$


In our case we want to show that the conic $D(x, y ; z)=0$ is an ellipse and that it has empty intersection with the set $x \geq 2$ and $y \geq 1$ for any $1 / 3<z<1$. Following the above definitions we can find the center and the semiaxes of $D(x+2, y+1, z)=0$ (all in terms of $z$ ) and check that $J>0, I \Delta<0$ and $I>0$ (the last condition implies that the interior has negative sign). All those inequalities are rational functions of $z$. To check the sign we multiply numerator and denominator of each function to get a polynomial in $z$. We find the roots (only polynomials up to fourth degree need to be considered) and check that all inequalities hold for $1 / 3<z<1$. Now we check if the distance between the center and the origin is larger than the square root of the sum of the squares of the semiaxes (the ellipse is contained in the circle with the same center and the radius larger than the longer semiaxes). Together with the condition that the center is in the third quadrant, this implies that the ellipse has empty intersection with the first quadrant for $1 / 3<z<1$ or, equivalently, that the theorem is true for $x \geq 2, y \geq 1$ and $1 / 3<z<1$.

Detailed calculations for all cases are included in Appendix 3 as a Mathematica script.

\section{Appendix 1}

The package TrigInt.m contains functions which are helpful to find variational upper bounds. All functions have short explanations available (use Fun in Mathematica). This package requires Mathematica 6.0. Although all functions are written for triangular domains, one could triangulate any polygon and still use the package.

The function TrigInt is equivalent to Integrate, but it is much faster for linear combinations of trigonometric functions. It is necessary due to a very slow integration of some trigonometric functions in Mathematica 5.1 and above.

Functions from this package are used to compute the variational upper bounds.

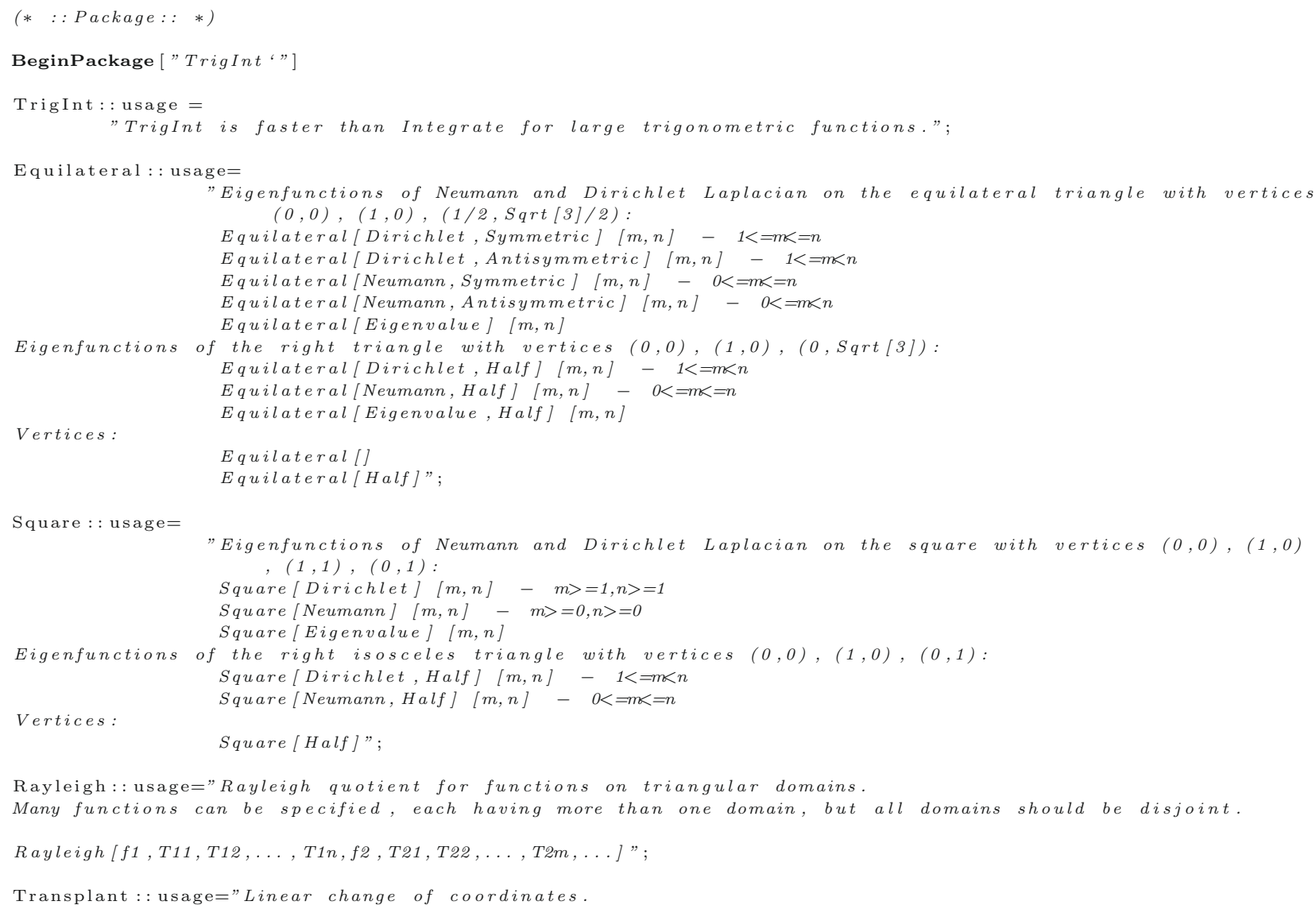


Transplant[Function, TargetTriangle, InitialTriangle]";

Limits:: usage="Generates limits for integration over a triangle with one side contained in the x-axis.";

Del: : usage="Gradient with respect to $x$ and $y$.";

Grad : : usage="Gradient with respect to $x$ and $y . "$;

Area: : usage="Area of a triangle with given vertices.",

Perimeter: : usage=" Perimeter of a polygon with given vertices."

$\mathrm{T}:$ : usage $=" T[a, b]-$ triangle with vertices $(0,0),(1,0)$ and $(a, b) " ;$

Begin [" "Private" "]

(* TrigInt *)

TrigInt $\left[\mathrm{f}_{-}, \mathrm{x}_{--}\right]:=$Expand $[\mathrm{TInt}[\operatorname{TrigReduce}[\mathrm{f}], \mathrm{x}]]$;

TInt $\left[\mathrm{f}_{-}, \mathrm{x}_{--}, \mathrm{y}_{\mathbf{-}}\right]:=\operatorname{TInt}[\operatorname{TInt}[\mathrm{f}, \mathrm{y}], \mathrm{x}]$;

TrigInt: : nomatch="No match for '1'. Integrate used.";

(* integrals *)

$\operatorname{psin}\left[\mathrm{n}_{-}\right]:=\operatorname{psin}[\mathrm{n}]=$ Evaluate $\left[\right.$ Integrate $\left.\left[\mathrm{y}^{\wedge} \mathrm{n} \operatorname{Sin}[\# 1+\# 2 \mathrm{y}],\{\mathrm{y}, \# 3, \# 4\}\right]\right] \& ;$

$p \cos \left[\mathrm{n}_{-}\right]:=\operatorname{pcos}[\mathrm{n}]=$ Evaluate $\left[\right.$ Integrate $\left.\left[\mathrm{y}^{\wedge} \mathrm{n} \operatorname{Cos}[\# 1+\# 2 \mathrm{y}],\{\mathrm{y}, \# 3, \# 4\}\right]\right] \&$;

$\operatorname{pp}\left[\mathrm{n}_{-}\right]:=\operatorname{pp}[\mathrm{n}]=$ Evaluate $\left[\right.$ Integrate $\left.\left[\mathrm{y}^{\wedge} \mathrm{n},\{\mathrm{y}, \# 1, \# 2\}\right]\right] \& ;$

(* substitutions *)

sub $\left[\mathrm{x}_{\mathbf{2}}, \mathrm{a}_{\mathbf{-}}, \mathrm{b}_{\mathbf{-}}\right]:=\{$

$\mathrm{X}_{\mathbf{-}} \cdot \mathrm{x}^{\wedge} \mathrm{n}_{\mathbf{-}} \cdot \operatorname{Sin}\left[\mathrm{A}_{-}+\mathrm{B}_{\mathbf{-}} \cdot \mathrm{x}\right] / ;$ FreeQ $[\{\mathrm{X}, \mathrm{A}, \mathrm{B}, \mathrm{n}\}, \mathrm{x}]:>(\mathrm{X} \operatorname{psin}[\mathrm{n}][\mathrm{A}, \mathrm{B}, \mathrm{a}, \mathrm{b}])$,

$\mathrm{X}_{-} . \mathrm{x}^{\wedge} \mathrm{n}_{-} \cdot \operatorname{Cos}\left[\mathrm{A}_{-}+\mathrm{B}_{-} . \mathrm{x}\right] / ;$ FreeQ $[\{\mathrm{X}, \mathrm{A}, \mathrm{B}, \mathrm{n}\}, \mathrm{x}]:>(\mathrm{X} \operatorname{pcos}[\mathrm{n}][\mathrm{A}, \mathrm{B}, \mathrm{a}, \mathrm{b}])$,

$\mathrm{X}_{\mathbf{2}} . \operatorname{Sin}\left[\mathrm{A}_{-} .+\mathrm{B}_{-} . \mathrm{X}\right] / ; \operatorname{FreeQ}[\{\mathrm{X}, \mathrm{A}, \mathrm{B}\}, \mathrm{X}]->(\mathrm{X} \operatorname{psin}[0][\mathrm{A}, \mathrm{B}, \mathrm{a}, \mathrm{b}])$,

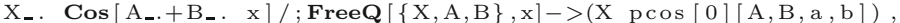

$\mathrm{X}_{-} . \quad \mathrm{x}^{\wedge} \mathrm{n}_{-} . / ;$FreeQ $[\{\mathrm{X}, \mathrm{n}\}, \mathrm{x}]:>(\mathrm{X} \operatorname{pp}[\mathrm{n}][\mathrm{a}, \mathrm{b}])$

$\mathrm{X}_{-} / ;$FreeQ $[\{\mathrm{X}\}, \mathrm{x}]->\mathrm{X}(\mathrm{b}-\mathrm{a})$,

$\mathrm{X}_{-}:>($Message $[$TrigInt : : nomatch, $\mathrm{X}] ;$ Integrate $[\mathrm{X},\{\mathrm{x}, \mathrm{a}, \mathrm{b}\}])$

\};

(* single integral *)

TInt $\left[\mathrm{f}_{-},\left\{\mathrm{x}_{-}, \mathrm{a}_{-}, \mathrm{b}_{-}\right\}\right]:=($

$\mathrm{ff}=\mathrm{f} / . \operatorname{Sin}\left[\mathrm{X}_{-}\right]:>\operatorname{Sin}[\operatorname{Collect}[\mathrm{X},\{\mathrm{x}\}, \operatorname{Simplify}]] / \operatorname{Cos}\left[\mathrm{X}_{-}\right]:>\operatorname{Cos}[\operatorname{Collect}[\mathrm{X},\{\mathrm{x}\}, \operatorname{Simplify}]]$;

$f f=$ Expand $[f f]$;

$\mathrm{ff}=\mathrm{ff}+\mathrm{f} 0+\mathrm{f} 1$

$\mathrm{ff}=$ Replace $[\mathrm{ff}, \mathrm{sub}[\mathrm{x}, \mathrm{a}, \mathrm{b}], 1]$;

$\mathrm{ff} / . \mathrm{f} 0->0 / . \mathrm{f} 1 \rightarrow 0$

) ;

* eigenfunctions *)

$\mathrm{h}=1$;

$\mathrm{r}=\mathrm{h} /(2 \operatorname{Sqrt}[3])$

$\mathrm{u}=\mathrm{r}-\mathrm{Global}$ ' $\mathrm{y}$

$\mathrm{v}=$ Sqrt [3] / $2($ Global ' $\mathrm{x}-\mathrm{h} / 2)+($ Global' $\mathrm{y}-\mathrm{r}) / 2$;

$\mathrm{w}=$ Sqrt [3] $/ 2(\mathrm{~h} / 2-$ Global' $\mathrm{x})+($ Global' $\mathrm{y}-\mathrm{r}) / 2$;

EqFun $\left[\mathrm{f}_{\mathbf{-}}, \mathrm{g}_{\mathbf{-}}\right]:=\mathrm{f}[\mathbf{P i}(-\# 1-\# 2)(\mathrm{u}+2 \mathrm{r}) /(3 \mathrm{r})] \mathrm{g}[\mathbf{P i}(\# 1-\# 2)(\mathrm{v}-\mathrm{w}) /(9 \mathrm{r})]+$

$\mathrm{f}[\mathbf{P i} \# 1(\mathrm{u}+2 \mathrm{r}) /(3 \mathrm{r})] \mathrm{g}[\mathbf{P i}(2 \# 2+\# 1)(\mathrm{v}-\mathrm{w}) /(9 \mathrm{r})]+$

$\mathrm{f}[\mathbf{P i} \# 2(\mathrm{u}+2 \mathrm{r}) /(3 \mathrm{r})] \mathrm{g}[\mathbf{P i}(-2 \# 1-\# 2)(\mathrm{v}-\mathrm{w}) /(9 \mathrm{r})]$

Equilateral [Global 'Neumann, Global'Symmetric]=Evaluate[Simplify [EqFun [Cos, Cos]]] \&;

Equilateral [Global 'Neumann, Global'Antisymmetric]=Evaluate [Simplify [EqFun [Cos, Sin]]] \&;

Equilateral [Global'Dirichlet, Global 'Symmetric]=Evaluate [Simplify [EqFun[Sin, Cos]]]\&;

Equilateral [Global 'Dirichlet, Global 'Antisymmetric]=Evaluate[Simplify [EqFun [Sin, Sin]]] \&;

Equilateral [Global 'Eigenvalue] =Evaluate $\left[4 / 27(\mathbf{P i} / \mathbf{r})^{\wedge} 2\left(\# 1^{\wedge} 2+\# 1 \# 2+\# 2^{\wedge} 2\right)\right] \&$;

Equilateral [Global 'Neumann, Global'Half]=Equilateral [Global 'Neumann, Global 'Symmetric]/. Global'x->(1-Global'x) $/ 2 /$. Global' $y \rightarrow$ Global'y/2//Simplify;

Equilateral [Global'Dirichlet, Global'Half]=Equilateral [Global 'Dirichlet, Global 'Antisymmetric]/.Global'x->(1Global'x)/2/. Global'y $\rightarrow$ Global'y/2//Simplify;

Equilateral [Global 'Eigenvalue, Global 'Half]=Evaluate[1/27(Pi/r)^2(\#1^2+\#1 \#2+\#2^2)]\&;

Equilateral []$=\mathrm{T}[1 / 2$, Sqrt $[3] / 2]$;

Equilateral [Global'Half $]=\mathrm{T}[0$, Sqrt [3] ];

Square [Global 'Dirichlet]=Evaluate [Sin[\#1 Pi Global'x] Sin [\#2 Pi Global'y]]\&;

Square [Global 'Neumann]=Evaluate [Cos[\#1 Pi Global'x] Cos[\#2 Pi Global'y]]\&;

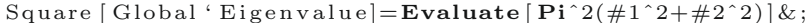

Square[Global'Dirichlet, Global'Half]=Evaluate[Sin[\#1 Pi Global'x] Sin[\#2 Pi Global'y]-(-1)^(\#1+\#2)Sin[\#2 Pi Global'x] Sin[\#1 Pi Global'y]]\&;

Square [Global'Neumann, Global'Half]=Evaluate [Cos[\#1 Pi Global'x] Cos[\#2 Pi Global'y]+(-1)^(\#1+\#2)Cos[\#2 Pi Global'x] Cos[\#1 Pi Global'y]]\&;

Square [Global'Half] $=\mathrm{T}[0,1]$

(* Transplantation *)

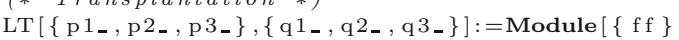




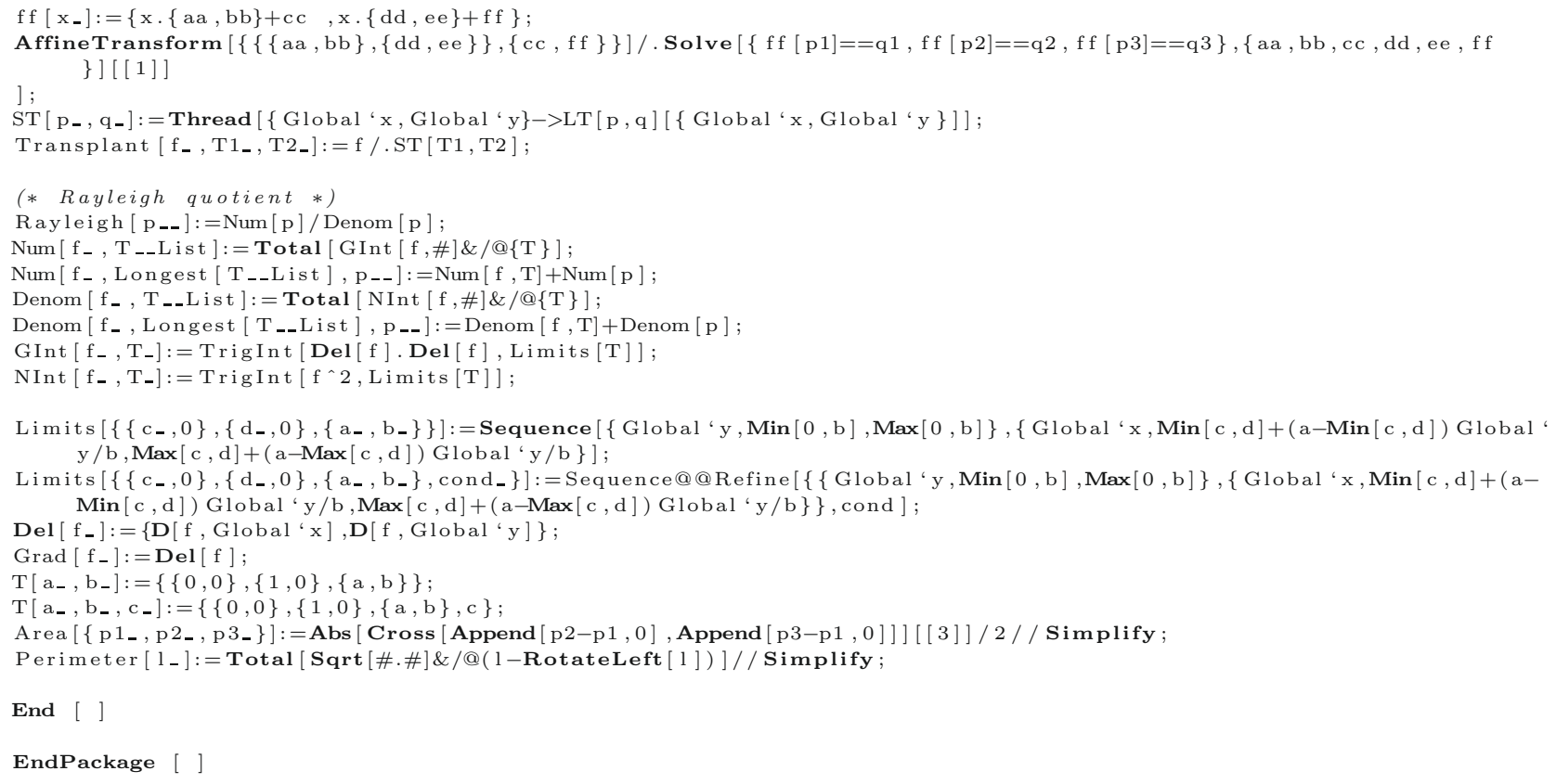

\section{ApPendix 2}

Here we present the script used in the proof of Theorem 3.2. The script outputs the plots of the numerical data, the fitted rational polynomials used in a linear combination and the results of the algorithm for solving polynomial inequalities.

It can be executed either inside Mathematica GUI or using a command line

MathKernel -run " all $=_{-} ;<<$script.m"

The parameter all should be either equal to a list of points or False. The first choice gives the values of the optimal variational bound at those points. The other value gives the proof of the theorem. This script requires the package from Appendix 1.

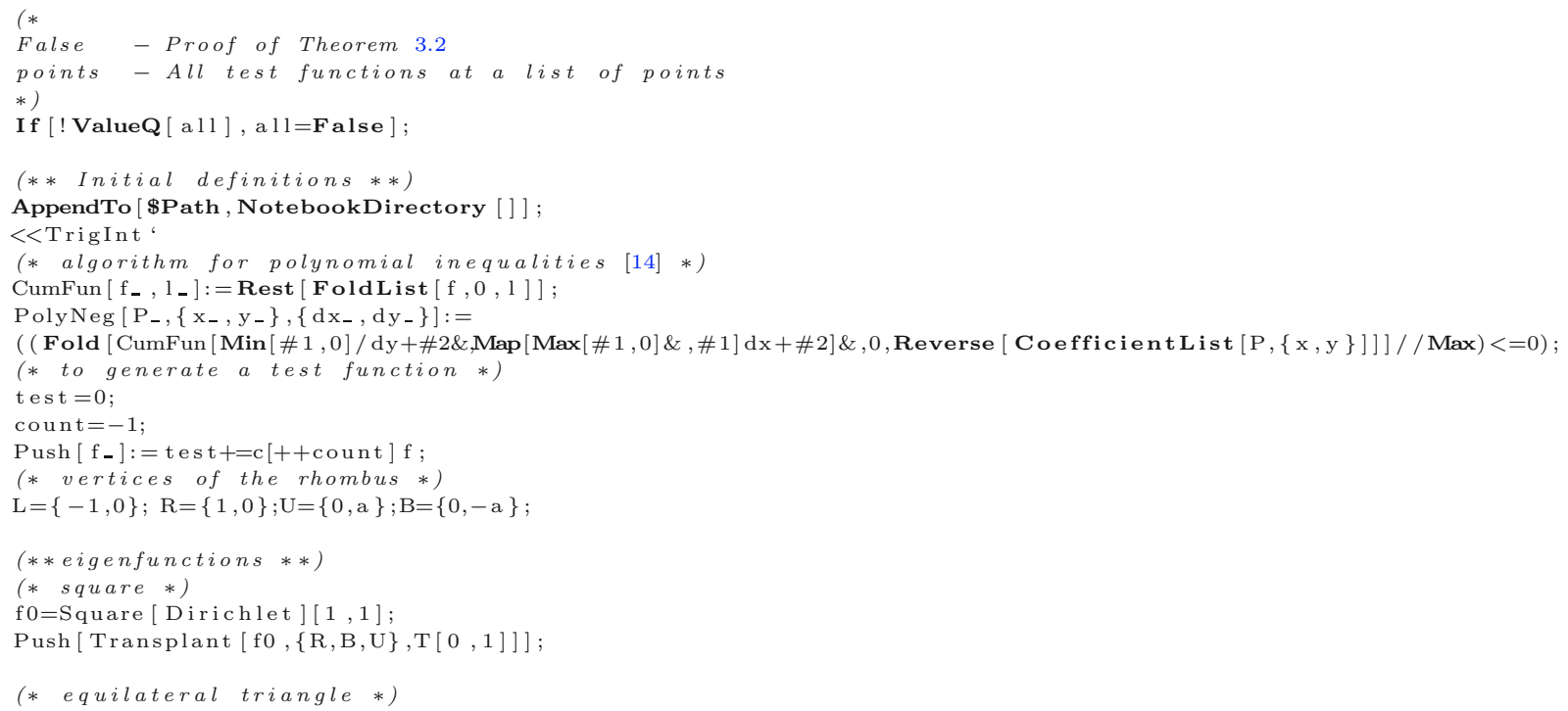


$\mathrm{f} 1=$ Equilateral [ Dirichlet, Symmetric] [ 1, 1];

$\mathrm{f} 2=$ Equilateral [Dirichlet, Antisymmetric] [1,2]; (* first eigenfunction into whole rhombus *) $\mathrm{T} 1=\mathrm{T}[1$, Sqrt $[3]] / 2$;

Push [ Transplant [f1 $,\{\mathrm{R}, \mathrm{B}, \mathrm{U}\}, \mathrm{T} 1]]$;

Push [ Transplant [f1, $\{\mathrm{R}, \mathrm{U}, \mathrm{B}\}, \mathrm{T} 1]]$;

Push [ Transplant [f1, \{U, L, R $\}, \mathrm{T} 1]]$;

Push [ Transplant [f1, \{U, R, L $\}, T 1]]$

(* first eigenfunction into halfs *)

T2=Equilateral [ ];

Push [ Transplant [f1, $\{\mathrm{L}, \mathrm{R}, \mathrm{U}\}, \mathrm{T} 2]]$;

Push [ Transplant [f1, $\{\mathrm{U}, \mathrm{B}, \mathrm{R}\}, \mathrm{T} 2]]$;

(* second eigenfunction into whole rhombus *)

$\mathrm{T} 3=\mathrm{T}[1 / 2$, Sqrt $[3] / 2] / 2$;

Push [ Transplant $[\mathrm{f} 2,\{\mathrm{R}, \mathrm{B}, \mathrm{U}\}, \mathrm{T} 3]]$;

Push [ Transplant [f2, $\{\mathrm{R}, \mathrm{U}, \mathrm{B}\}, \mathrm{T} 3]]$;

Push [ Transplant [f2, \{U, L, R $\}, T 3]]$;

Push [ Transplant [f2, $\{\mathrm{U}, \mathrm{R}, \mathrm{L}\}, \mathrm{T} 3]]$;

If $[$ a 1$]=!=$ False

$\mathrm{T} 6=\{\{1 / 2$, Sqrt $[3] / 2\},\{1 / 2,0\},\{1 / 4$, Sqrt $[3] / 4\}\} ;$

Push [ Transplant [f $2,\{\mathrm{R}, \mathrm{B}, \mathrm{U}\}, \mathrm{T} 6]]$;

Push [ Transplant [f2, $\{\mathrm{R}, \mathrm{U}, \mathrm{B}\}, \mathrm{T} 6]]$;

Push [ Transplant [f2, $\{\mathrm{U}, \mathrm{L}, \mathrm{R}\}, \mathrm{T} 6]]$;

Push [Transplant [f2, $\{\mathrm{U}, \mathrm{R}, \mathrm{L}\}, \mathrm{T} 6]]$;

$\mathrm{T} 5=\mathrm{T}[1 / 4$, Sqrt $[3] / 4] / 2$;

Push [ Transplant [ f2, $\{\mathrm{R}, \mathrm{B}, \mathrm{U}\}, \mathrm{T} 5]]$;

Push [ Transplant [f2, $\{\mathrm{R}, \mathrm{U}, \mathrm{B}\}, \mathrm{T} 5]]$;

Push [ Transplant [f $2,\{\mathrm{U}, \mathrm{L}, \mathrm{R}\}, \mathrm{T} 5]]$;

Push [Transplant [f2, \{U, R, L $\}, T 5]] ;$

$\mathrm{T} 7=\{\{1 / 2$, Sqrt $[3] / 2\},\{1 / 2,0\},\{1 / 8$, Sqrt $[3] / 8\}\} ;$

Push [ Transplant [ f2, $\{\mathrm{R}, \mathrm{B}, \mathrm{U}\}, \mathrm{T} 7]]$;

Push [ Transplant $[\mathrm{f} 2,\{\mathrm{R}, \mathrm{U}, \mathrm{B}\}, \mathrm{T} 7]]$;

Push [ Transplant [f2, $\{\mathrm{U}, \mathrm{L}, \mathrm{R}\}, \mathrm{T} 7]]$;

Push [ Transplant [f $2,\{\mathrm{U}, \mathrm{R}, \mathrm{L}\}, \mathrm{T} 7]]$

(* second eigenfunction into halves *)

Push [ Transplant [f2, $\{\mathrm{U}, \mathrm{L}, \mathrm{R}\}, \mathrm{T} 1]]$;

Push [Transplant $[\mathrm{f} 2,\{\mathrm{U}, \mathrm{R}, \mathrm{L}\}, \mathrm{T} 1]]$;

Push [Transplant $[\mathrm{f} 2,\{\mathrm{~L}, \mathrm{U}, \mathrm{R}\}, \mathrm{T} 1]]$;

Push [ Transplant [f2, $\{\mathrm{L}, \mathrm{R}, \mathrm{U}\}, \mathrm{T} 1]]$;

Push [ Transplant [f2, $\{\mathrm{R}, \mathrm{L}, \mathrm{U}\}, \mathrm{T} 1]]$;

Push [ Transplant [f2, $\{\mathrm{R}, \mathrm{U}, \mathrm{L}\}, \mathrm{T} 1]]$;

Push [ Transplant [f2, $\{\mathrm{U}, \mathrm{B}, \mathrm{R}\}, \mathrm{T} 1]]$;

Push [ Transplant [f2, $\{\mathrm{U}, \mathrm{R}, \mathrm{B}\}, \mathrm{T} 1]]$;

Push [ Transplant [f2, $\{\mathrm{B}, \mathrm{U}, \mathrm{R}\}, \mathrm{T} 1]]$;

Push [ Transplant [f2, $\{\mathrm{B}, \mathrm{R}, \mathrm{U}\}, \mathrm{T} 1]]$;

Push [ Transplant [ f2, $\{\mathrm{R}, \mathrm{B}, \mathrm{U}\}, \mathrm{T} 1]]$;

Push [ Transplant $[\mathrm{f} 2,\{\mathrm{R}, \mathrm{U}, \mathrm{B}\}, \mathrm{T} 1]]$; ];

(* right isosceles triangle *)

$\mathrm{f} 3=$ Square [ Dirichlet, Half ] [1,2];

(* first eigenfunction into whole rhombus *)

$\mathrm{T} 4=\{\{0,0\},\{0,1\},\{1 / 2,1 / 2\}\} ;$

Push [ Transplant [ f $3,\{\mathrm{U}, \mathrm{R}, \mathrm{B}\}, \mathrm{T} 4]]$;

Push [ Transplant $[\mathrm{f} 3,\{\mathrm{~B}, \mathrm{R}, \mathrm{U}\}, \mathrm{T} 4]]$;

Push [Transplant $[\mathrm{f} 3,\{\mathrm{~L}, \mathrm{U}, \mathrm{R}\}, \mathrm{T} 4]]$;

Push [ Transplant [f $3,\{\mathrm{R}, \mathrm{U}, \mathrm{L}\}, \mathrm{T} 4]]$

(* first eigenfunction into halfs *)

Push [ Transplant [f $3,\{\mathrm{R}, \mathrm{U}, \mathrm{B}\}, \mathrm{T}[0,1]]]$;

Push [ Transplant [f $3,\{\mathrm{U}, \mathrm{L}, \mathrm{R}\}, \mathrm{T}[0,1]]]$;

If $[$ a 1$]=!=$ False

Push [ Transplant [f $3,\{\mathrm{~B}, \mathrm{R}, \mathrm{U}\}, \mathrm{T}[0,1]]]$;

Push [ Transplant [f $3,\{\mathrm{U}, \mathrm{B}, \mathrm{R}\}, \mathrm{T}[0,1]]]$

Push [ Transplant [f $3,\{\mathrm{R}, \mathrm{U}, \mathrm{L}\}, \mathrm{T}[0,1]]]$

Push [ Transplant $[\mathrm{f} 3,\{\mathrm{~L}, \mathrm{R}, \mathrm{U}\}, \mathrm{T}[0,1]]]$

];

(* Rayleigh quotient on the upper right quarter of a rhombus *) bound=R ayleigh [ test, $\mathrm{T}[0, \mathrm{a}, \mathrm{a}>0]]$;

If $[$ a $11===$ False,

(** proof of Theorem $3.2 * *$ )

(* generate 1000 points *)

$\mathrm{n}=1000$ 


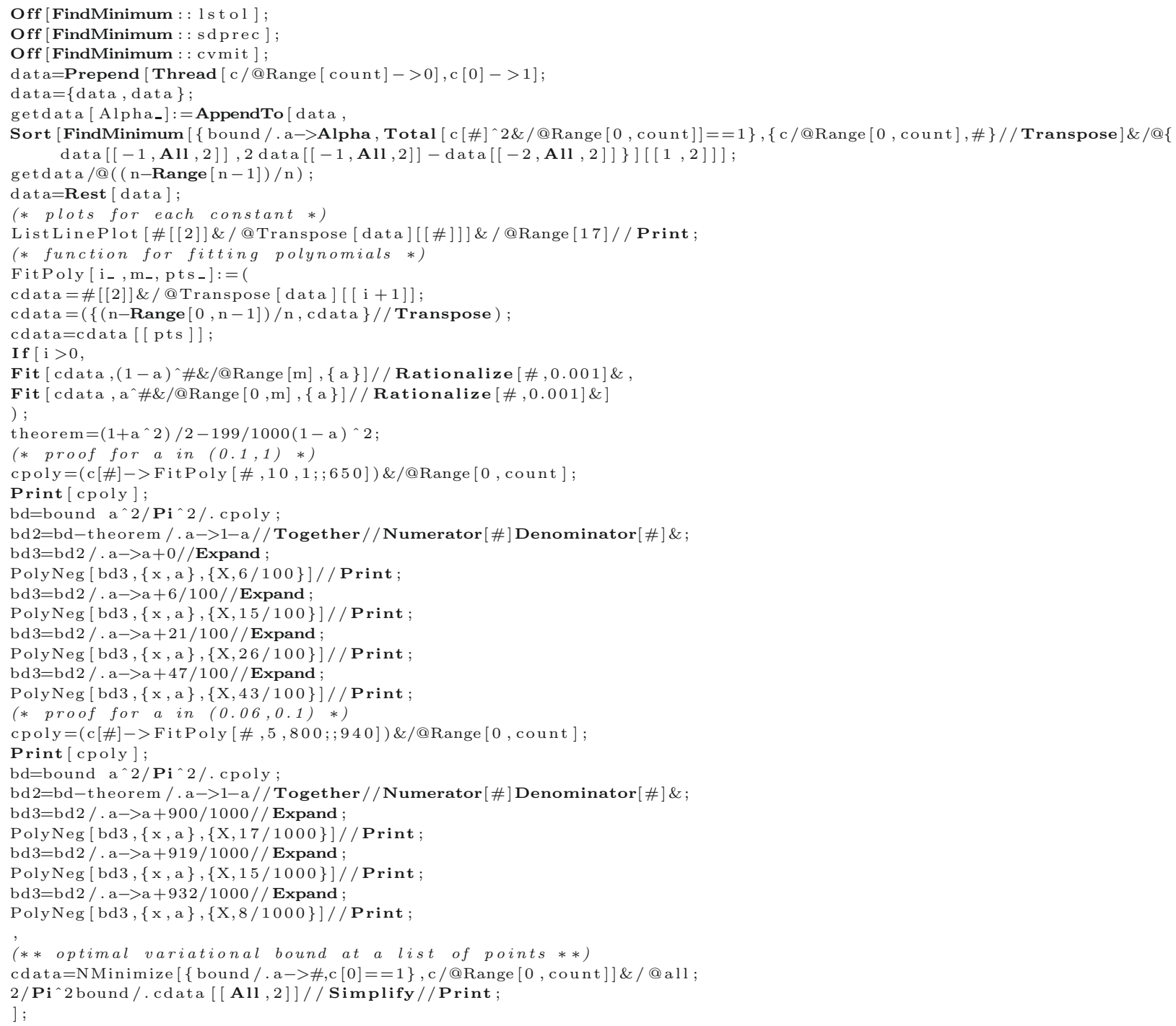

\section{ApPendix 3}

The script from this appendix performs all the calculations needed in the proof of the upper bound from Theorem 1.1. It can be executed analogously to the previous script except that there is no parameter.

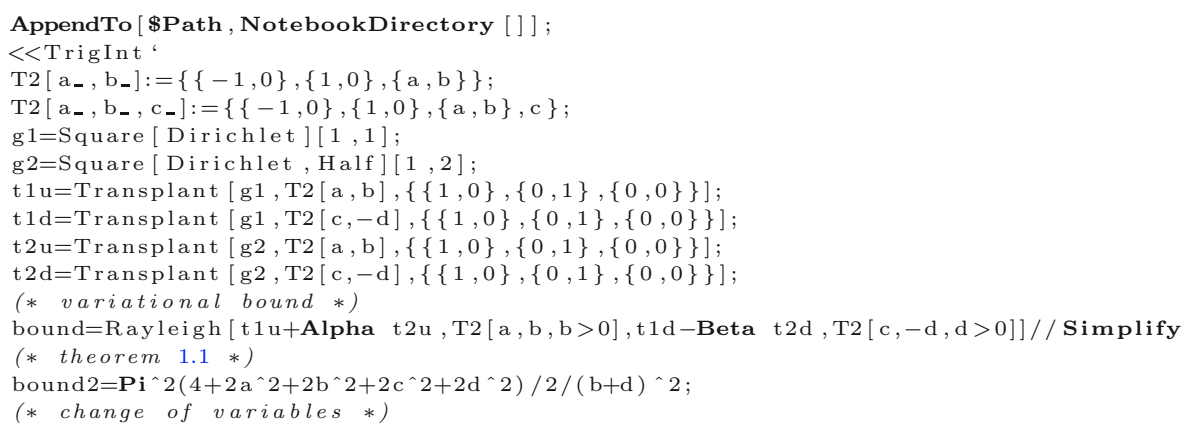




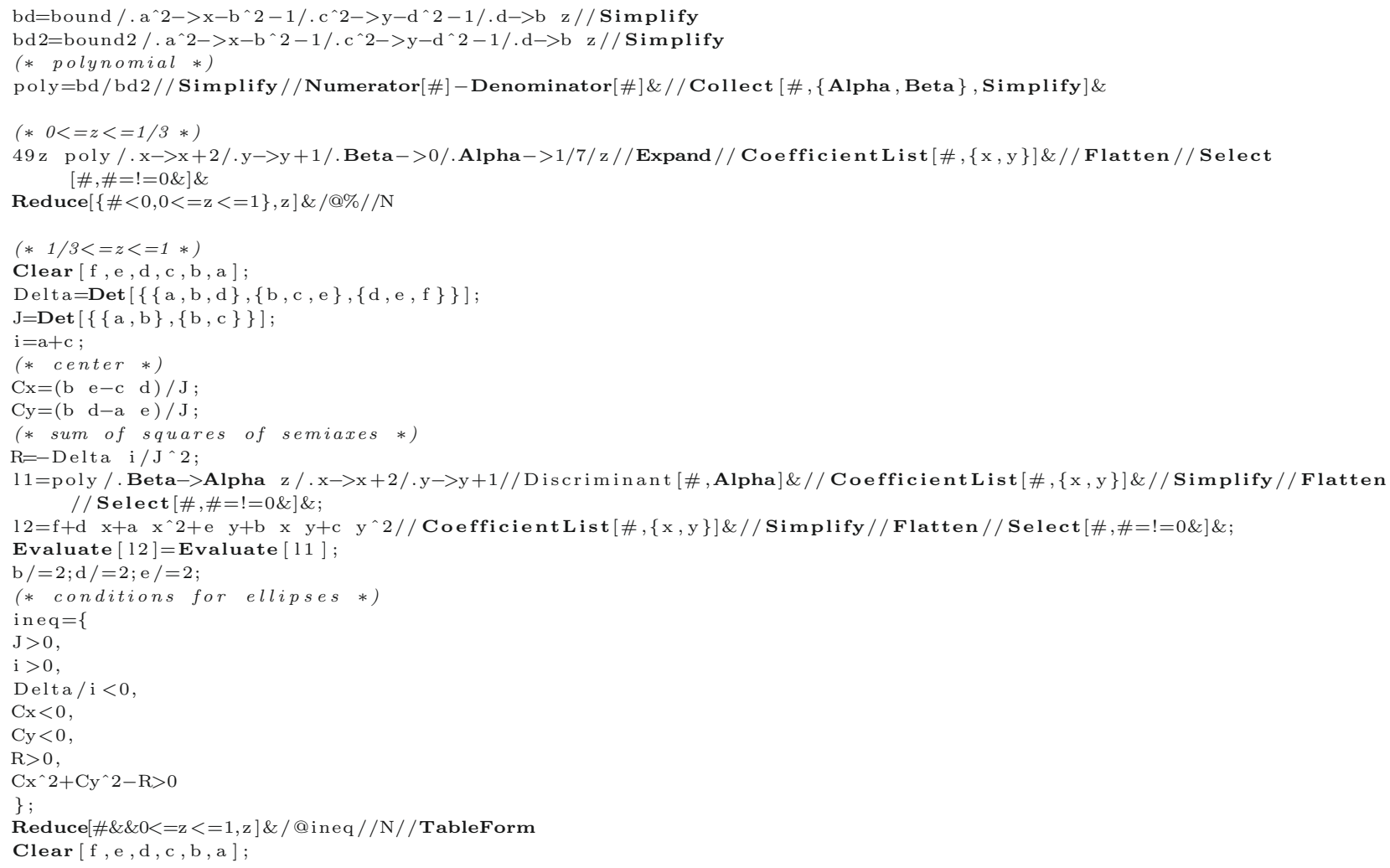

\section{REFERENCES}

[1] P. Antunes and P. Freitas, New bounds for the principal Dirichlet eigenvalue of planar regions. Experiment. Math. 15 (2006) 333-342.

[2] P. Antunes and P. Freitas, A numerical study of the spectral gap. J. Phys. A 41 (2008) 055201.

[3] D. Borisov and P. Freitas, Singular asymptotic expansions for Dirichlet eigenvalues and eigenfunctions on thin planar domains. Ann. Inst. H. Poincaré Anal. Non Linéaire 26 (2009) 547-560.

[4] P. Freitas, Upper and lower bounds for the first Dirichlet eigenvalue of a triangle. Proc. Amer. Math. Soc. 134 (2006) $2083-2089$.

[5] P. Freitas, Precise bounds and asymptotics for the first Dirichlet eigenvalue of triangles and rhombi. J. Funct. Anal. 251 (2007) 376-398.

[6] J. Hersch, Constraintes rectilignes parallèles et valeurs propres de membranes vibrantes. Z. Angew. Math. Phys. 17 (1966) $457-460$.

[7] W. Hooker and M.H. Protter, Bounds for the first eigenvalue of a rhombic membrane. J. Math. Phys. 39 (1960/1961) 18-34.

[8] E. Makai, On the principal frequency of a membrane and the torsional rigidity of a beam, in Studies in mathematical analysis and related topics, Essays in honor of George Pólya, Stanford Univ. Press, Stanford (1962) 227-231.

[9] P.J. Méndez-Hernández, Brascamp-Lieb-Luttinger inequalities for convex domains of finite inradius. Duke Math. J. 113 (2002) 93-131.

[10] G. Pólya and G. Szegö, Isoperimetric inequalities in mathematical physics, Annals of Mathematical Studies 27. Princeton University Press, Princeton (1951).

[11] M.H. Protter, A lower bound for the fundamental frequency of a convex region. Proc. Amer. Math. Soc. 81 (1981) 65-70.

[12] C.K. Qu and R. Wong, "Best possible" upper and lower bounds for the zeros of the Bessel fuction $J_{v}(x)$. Trans. Amer. Math. Soc. 351 (1999) 2833-2859.

[13] B. Siudeja, Sharp bounds for eigenvalues of triangles. Michigan Math. J. 55 (2007) 243-254.

[14] B. Siudeja, Isoperimetric inequalities for eigenvalues of triangles. Ind. Univ. Math. J. (to appear). 